\title{
Taxonomic revision of Phyllanthus (Phyllanthaceae) in Madagascar and the Comoro Islands IV: subgenus Anisonemoides section Pseudogomphidium and incertae sedis
}

\author{
Hélène Ralimanana ${ }^{1}$ (D) \& Stuart Cable ${ }^{2}$
}

Summary. This paper is the fourth part of the taxonomic revision of the genus Phyllanthus in Madagascar and the Comoro Islands. Here we treat Pseudogomphidium Ralim. \& Cable sect. nov. consisting of nine species that we misplaced in the subgenus Gomphidium (Baill.) G.L.Webster in our previous paper. Two species are new to science: Phyllanthus razakamalalae Ralim. \& Cable, sp. nov. and P. ankirindrensis Ralim. \& Cable, sp. nov. We also treat three unplaced species: P. analamerae Leandri, P. coluteoides Müll.Arg. and P. vergens Baill. An identification key, descriptions, distribution maps and IUCN Red List assessments are provided.

Key Words. IUCN Red List, Phyllanthus ankirindrensis, Phyllanthus razakamalalae, taxonomy.

\section{Introduction}

Phyllanthus is one of the largest genera of plants known from Madagascar with 52 native species. It is often neglected by botanists because of complex taxonomy and difficulty in identifying species due to the small flowers.

The revision of Malagasy Phyllanthus by the lead author delimits nine other subgenera as detailed in three papers prior to this one (Ralimanana \& Hoffmann 2011; Ralimanana et al. 2013, and Ralimanana \& Hoffmann 2014). The first gave a general synopsis of the genus in the region and treated four subgenera in detail: Isocladus G.L.Webster, Betsileani (Jean F.Brunel) Ralim. \& Petra Hoffm., Kirganelia (A.Juss.) G.L.Webster and Tenellanthus Jean F.Brunel (Ralimanana \& Hoffmann 2011). The second paper treated subgenera: Anisonemoides (Jean F.Brunel) Ralim. \& Petra Hoffm. and Menarda (Comm. ex A.Juss.) Ralim. \& Petra Hoffm. (Ralimanana \& Hoffmann 2014). The third paper dealt with subgenera: Swartziani (G.L.Webster) Ralim. \& Petra Hoffm., Afroswartziani Ralim. \& Petra Hoffm. and Emblica (Gaertn.) G.L.Webster (Ralimanana et al. 2013). This paper is the last in the series of the systematic revision of Phyllanthus L. in Madagascar and the Comoro Islands.

Phyllanthus is the most diverse genus in the family Phyllanthaceae (Hoffman et al. 2006). Floral characters are very important in species and subgenus identification, but species can be morphologically variable, and many characters are shared with closely related genera (e.g. Meineckia Baill. and Flueggea Willd.). The generic delimitation of Phyllanthus has long been debated in the literature and classification of species remains a challenge. Presently Phyllanthus is broadly circumscribed to contain several segregate genera including Glochidion J.R.Forst. \& G.Forst., Breynia J.R.Forst. \& G.Forst. and Sauropus Blume, and is a member of tribe Phyllantheae Dumort. (Hoffmann et al. 2006), the largest and most difficult group in Phyllanthaceae sensu APG (Hoffmann \& McPherson 2003; Hoffmann et al. 2006), formerly Euphorbiaceae subfamily Phyllanthoideae.

Some Malagasy Phyllanthus were originally described by Leandri (1934 - 1957) under Glochidion. In the Flore de Madagascar et des Comores, Leandri (1958) recognised seven species: Glochidion ankaratrae Leandri, G. humbertii Leandri, G. lichenisilvae Leandri, G. marojejiensis Leandri, G. oreichtitum Leandri, G. perrieri Leandri, and G. sambiranensis Leandri.

In his treatment of Phyllanthus of intertropical Africa, Brunel (1987) indicated the need to create a new section to accommodate the Malagasy Glochidion species sensu Leandri (1958), based on their pollen characters and floral morphology. After reexamination and study of specimens held in the Paris Herbarium and based on disc and seed characters, Hoffmann \& McPherson (2003) moved these species to Phyllanthus. Although molecular research by Kathriarachchi et al. (2006) demonstrated that Glochidion is nested within Phyllanthus. 
Broad understanding of pollination biology of Phyllantheae was not known before the investigation of Kato et al. (2003), Kato \& Kawakita (2017b) and Kawakita \& Kato (2004a, b; 2009). Their results showed that some species belonging to subgenus Gomphidium are nocturnally pollinated by a speciesspecific Epicephala moth whose, larvae feed on the seed, collected and transported pollen between flowers (Kawakita \& Kato 2006; Kawakita \& Kato 2009). Within the investigation of the origin of Phyllantheae - Epicephala mutualism, they reconstructed phylogeny for 46 species of Phyllanthaceae among them 22 Epicephala-pollinated including Phyllanthus marojejiensis (Leandri) Petra Hoffm. \& McPherson and $P$. humbertii (Leandri) Petra Hoffm. \& McPherson (both from Madagascar). This recent molecular analysis brought new updated information on the phylogenetic position of Madagascar Phyllanthus in respect to the species from New Caledonia, the centre of distribution of the subgenus Gomphidium. It showed that species pollinated by Epicephala were not monophyletic, the species from Madagascar are divergent from the clade containing the New Caledonian species and fall into a separate clade. Based on their findings, Kato \& Kawakita (2017a) stated that $P$. humbertii and $P$. marojejiensis are closely related to subgenera Betsileani and Anisonemoides.

Subgenus Betsileani has non-phyllanthoid branching (all branches are equivalent), has three, entirely free stamens and pollen are perisyncoloprate. Entirely fused stamens of Phyllanthus bernierianus Baill. were noted as atypical of subgen. Anisonemoides (Ralimanana \& Hoffmann 2014). However, the molecular data (Kathriarachchi et al. 2006) showed that P. bernieranus and $P$. vakinankaratrae Leandri (Anisonemoides with $5-6$ free stamens) form a strongly supported clade (BP 99).

Morphologically the taxa treated here have phyllanthoid branching, 3 - 6 free or fused stamens and dehiscent fruits which are similar to the taxa in subgen. Anisonemoides. Brunel (1987) found that Phyllanthus sambiranensis Leandri and P. oreichtitus had both tricopolrate pollen with macromicroreticulate exine which is similar to subgen. Anisonemoides (stephanocolporate pollen, rugulate exine in subgen. Gomphidium (Webster 1994)). Therefore, based on the morphological pattern, floral and pollen characters and genetic phylogeny, we here create a new section Pseudogomphidium Ralim. \& Cable sect. nov. in subgen. Anisonemoides to accommodate the species grouped in subgen. Gomphidium sensu Ralimanana \& Hoffmann (2011). It comprises ten species which are endemic to Madagascar and includes $P$. bernierianus treated in the previous paper. Two species $P$. razakamalalae Ralim. \& Cable and $P$. ankirindrensis Ralim. \& Cable are new to science and described in this paper.
We also present three unplaced species: Phyllanthus analamerae Leandri, $P$. coluteoides Baill. and P. vergens Baill.

\section{Materials and Methods}

This revision is based on herbarium material and field observations from throughout Madagascar. Herbarium specimens were examined at Kew (K), Paris (P) and in Madagascar (TAN and TEF). The descriptions are mostly based on dried specimens or softened plant parts from herbarium material. Distribution maps and conservation assessments were obtained using GIS (Arcview 3.3) and by applying IUCN criteria (IUCN 2012).

The floristic domains follow Humbert (1965). Information about habitat was obtained from field studies or from the field notes of other botanists.

\section{Taxonomic treatment}

1. Phyllanthus subgen. Anisonemoides (Jean F.Brunel) Ralim. EF Petra Hoffm. sect. Pseudogomphidium Ralim $\mathcal{E}$ Cable, sect. nov. Type: Phyllanthus marojejiensis (Leandri) Petra Hoffm. \& McPherson.

http://www.ipni.org/urn:lsid:ipni.org:names:77213109-1

Monoecious shrubs or subshrubs with pinnatiform phyllanthoid branching (main branches with unlimited growth and leaves reduced to scales (cataphylls), the lateral (plagiotropic) with limited growth, deciduous, leafy and floriferous). Plagiotropic branches solitary. Leaves alternate. Inflorescences axillary, unisexual or bisexual cymules, 1 - 4flowered. Tepals $5(-6)$. Disc glands free or absent in male flowers, lobed, extremely reduced or absent in female flowers. Stamens $3-5$ (- 6); filaments free, partially or entirely fused into a column; anthers free or fused, oblong, basifixed, dehiscing longitudinally, apiculate or not apiculate; pollen tricolporate, macromicroreticulate exine. Ovary 3-locular, smooth or slightly rugose; styles free, deltoid, slightly bifid or entire; stigmas obtuse or acute. Fruits dehiscent; tepals persistent, sometimes accrescent in fruit; styles persistent; seeds 2 or sometimes 1 per locule, smooth or thinly striated longitudinally.

ReCOGNITION. Section Pseudogomphidium is similar to subgen. Gomphidium sect. Gomphidium and subgen. Anisonemoides sect. Anisonemoides because of the phyllanthoid branching, disc glands in male flowers, and trilocular dehiscent fruits, but differs from sect. Gomphidium in the $5(-6)$ sepals (vs 6) and tricolporate pollen (vs trisyncolporate); differs from sect. Anisonemoides in the clustered bracts (vs not clustered), 
the oblong anthers (vs globose or ovoid), relatively reduced or disc absent in female flowers (vs lobed), the free, entire or slightly bifid style (vs fused and bifid).

Key to the species of Phyllanthus subgen. Anisonemoides sect. Pseudogomphidium

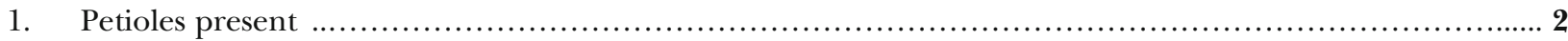

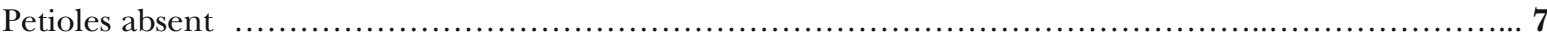

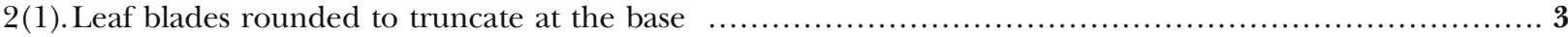

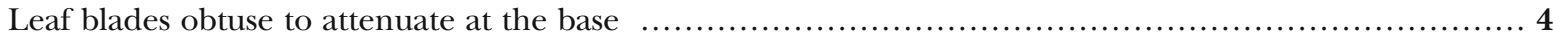

3(2). Plagiotropic branches terete. Leaf blades acute at the apex. Inflorescence unisexual. Pedicels $6-9 \mathrm{~mm}$ in male flowers, $8-11 \mathrm{~mm}$ in female flowers. Disc glands absent in male flowers. Anthers apiculate. Female

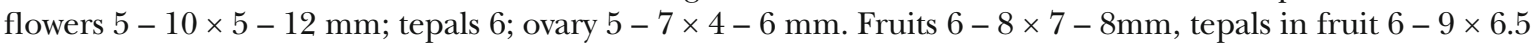
$-8 \mathrm{~mm}$. Seeds thinly striated longitudinally

1. P. ankirindrensis Plagiotropic branches compressed. Leaf blades acuminate at the apex. Inflorescence bisexual. Pedicels $2-4 \mathrm{~mm}$ in male flowers, $18-25 \mathrm{~mm}$ in female flowers. Disc glands present in male flowers. Anthers not apiculate. Female

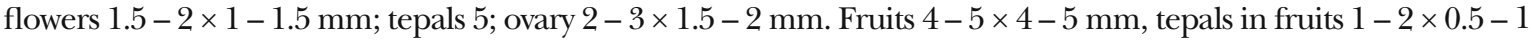
mm. Seeds smooth

2. P. humbertii

4(3). Leaf blades obovate to oblong. Inflorescence bisexual or unisexual. Filaments entirely fused; anthers not apiculate. Female flowers $1-1.3 \times 0.5-1 \mathrm{~mm}$; ovary $0.8-1 \times 0.8-1 \mathrm{~mm}$. Fruits c. $2 \times 1.8 \mathrm{~mm}$. Seeds $1-$ $1.2 \times 0.7-1$.

3. P. razakamalalae Leaf blades ovate, elliptic, or subrhomboid. Inflorescence always unisexual. Filaments free or the inner fused up to $1 / 3$ their length; anthers apiculate. Female flowers $2-5 \times 2-3.5 \mathrm{~mm}$; ovary $1.7-2.6 \times 1.7-2.6 \mathrm{~mm}$. Fruits $2.8-4 \times 3-4.5 \mathrm{~mm}$. Seeds $1.8-2.5 \times 1.7-2.4 \mathrm{~mm}$

5(4). Plagiotropic branches pubescent. Bracts not clustered in male inflorescences. Stamens 6. Female flowers pedicels $7-20 \mathrm{~mm}$ long. Fruiting pedicels $8-20 \mathrm{~mm}$ long $\ldots \ldots \ldots \ldots \ldots \ldots \ldots \ldots \ldots$ 4. P. oreichtitus Plagiotropic branches glabrous. Bracts in cluster of 3-6 mainly sterile bracts in male inflorescences. Stamens

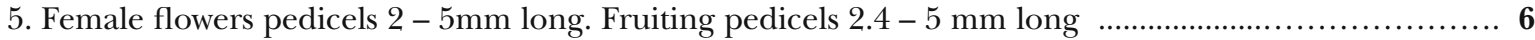

6(5). Leaf blades symmetrical, flat; midvein flattened adaxially, prominent abaxially. Tepals $3-4 \times 1.5-2 \mathrm{~mm}$ in male flowers, $4-5 \times 2-5 \mathrm{~mm}$ in female flowers, and $4.2-5 \times 2-5.3 \mathrm{~mm}$ in fruits $\ldots \ldots \ldots \ldots$.... P. perrieri Leaf blades asymmetrical, margin revolute; midvein prominent adaxially and abaxially. Tepals $1.5-2.2 \times 0.8-2 \mathrm{~mm}$ in male flowers, $2-2.2 \times 1-1.2 \mathrm{~mm}$ in female flowers, and $2.2-2.8 \times 1-1.2 \mathrm{~mm}$ in fruits ....... 6. P. sambiranensis

7(1). Leaf blades spatulate falcate, $0.5-1.5 \mathrm{~mm}$ wide, concave abaxially; secondary veins indistinct adaxially and abaxially 7. P. marojejiensis

Leaf blades $2.5-4.5 \mathrm{~mm}$ wide, flat; secondary veins flattened or prominent abaxially $\ldots \ldots \ldots \ldots \ldots \ldots \ldots .8$ $8(7)$. Leaf blades subcoriaceous to coriaceous. Stamen with a central apiculum. Tepals $5,2.8-3.5 \times 1.4-1.6 \mathrm{~mm}$ in female flowers. Disc annular pentagonal. Ovary $1.8-2 \times$ c. $2 \mathrm{~mm} \quad \ldots \ldots \ldots \ldots \ldots \ldots \ldots \ldots \ldots$. 8. P. lichenisilvae Leaf blades membranous to chartaceous. Anthers not apiculate and different lengths. Tepals $6,1.3-1.5 \times 1-$ $1.2 \mathrm{~mm}$ in female flowers. Disc minute or absent. Ovary $1-1.3 \times 1-1.4 \mathrm{~mm}$

9. P. ankaratrae

1. Phyllanthus ankirindrensis Ralim. E Cable, sp. nov. Type: Madagascar. Toamasina, Maroantsetra, summit of Ankirindro massif, $15^{\circ} 18^{\prime} 08^{\prime \prime} \mathrm{S}, 4^{\circ} 33^{\prime} 04^{\prime \prime} \mathrm{E}$, alt. 671 m, 1 Feb. 1999, Schatz 3916 (holotype P!; isotype MO).

http:/ /www.ipni.org/urn:lsid:ipni.org:names:77213110-1

Monoecious shrubs, to $1.50 \mathrm{~m}$ high. Branching pinnatiform, orthotropic branches terete, glabrous. Cataphylls and cataphyllary stipules caducous. Plagiotropic branches solitary, terete, $6-12 \mathrm{~cm}$ long, $1-1.8 \mathrm{~mm}$ in diam., smooth, glabrous. Stipules caducous. Leaves 3 6 per branch. Internodes $5-17 \mathrm{~mm}$ long. Petioles terete, $2-3 \times 1-1.5 \mathrm{~mm}$, glabrous. Leaf blades ovate, $30-60 \times$
$20-30 \mathrm{~mm}$, rounded to truncate at the base, acute at the apex, chartaceous to subcoriaceous, glabrous on both sides; midvein flattened adaxially, prominent abaxially; secondary veins $2-4$ pairs, flattened adaxially, prominent abaxially. Inflorescence unisexual, consisting of one male or one female flower, male and female inflorescences on different branches. Bracts triangular, 0.7 - $1 \times$ $0.5-0.7 \mathrm{~mm}$, entire, glabrous. Male flowers $4-6 \times 3-4$ mm. Pedicels $6-9 \times 0.4-1 \mathrm{~mm}$, glabrous. Tepals 5, unequal, oblong, $3-5 \times 2.5-4 \mathrm{~mm}$, obtuse, coriaceous, glabrous on both sides, veins unbranched. Disc absent. Stamens 5, entirely fused into a column; column terete, widened at the base, $1.5-2 \times 0.5-0.8 \mathrm{~mm}$; anthers fused, oblong, apiculate, $1-1.5 \times 0.2-0.4 \mathrm{~mm}$. Female 
flowers 5-10×5-12 mm. Pedicels $8-11 \times 0.5-2 \mathrm{~mm}$, glabrous. Tepals 6 , unequal, on two whorls (outer smaller, inner bigger), ovate, $5-7 \times 6-7 \mathrm{~mm}$, rounded, subcoriaceous, glabrous on both sides, margin entire, veins branched. Disc absent or lobed, thin, $0.1-0.2 \mathrm{~mm}$ thick. Ovary 3-locular, ovoid, 5-7×4-6 mm, smooth or slightly rugose, glabrous. Styles free, deltoid, slightly bifid at the apex, c. $1 \mathrm{~mm}$ long; stigmas obtuse. Fruits ovoid, $6-$ $8 \times 7-8 \mathrm{~mm}$, exocarp thick c. $2 \mathrm{~mm}$; fruiting pedicels $8-$ $11 \times$ c. $2 \mathrm{~mm}$, glabrous. Tepals persistent, accrescent, 6 $9 \times 6.5-8 \mathrm{~mm}$; columella c. $2-3 \mathrm{~mm}$. Seeds $2-4 \times 2 \mathrm{~mm}$, thinly striated longitudinally. Fig. 1.

RECOGNITION. Phyllanthus ankirindrensis is similar to $P$. humbertii by the presence of a petiole, leaf blades rounded to truncate at the base and the stamens fused into a column, but differs from $P$. humbertii by leaf blades acute at the apex (vs acuminate), the inflorescence unisexual (vs bisexual), the disc glands absent in male flower (vs present), the 5 stamens (vs 3), female flowers size, $5-10 \times 5-11 \mathrm{~mm}$ (vs $1.5-2 \times 1-1.5$ $\mathrm{mm}$ ), the 6 tepals (vs 5 ), the ovary $5-7 \times 4-6 \mathrm{~mm}$ (vs $2-3 \times 1.5-2 \mathrm{~mm}$ ), the fruits ovoid (vs globose), $6-8$ $\times 7-8 \mathrm{~mm}($ vs $4-5 \times 4-5 \mathrm{~mm})$ and the size of tepals in fruit $6-9 \times 6.5-8 \mathrm{~mm}$ (vs $1-2 \times 0.5-1 \mathrm{~mm}$ ).

DISTRIBUTION. Endemic to Madagascar. Eastern floristic domain. Map 1.

SPECIMENS EXAMINED. MADAGASCAR. Toamasina: Maroantsetra, sommet du massif d'Ankirindro, $15^{\circ} 17^{\prime} 37^{\prime \prime S}, 49^{\circ} 33^{\prime} 10^{\prime \prime} \mathrm{E}$, alt. 640 m, Jan. 2003, Antilahimena et al. 1668 (K, MO); Maroantsetra, Ambinanitelo, Marovovonana, $15^{\circ} 18^{\prime} 41^{\prime \prime} \mathrm{S}, 4^{\circ} 25^{\prime} 28^{\prime \prime}$, alt. $981 \mathrm{~m}$, Sept. 2004, Antilahimena et al. 2689 (K, MO); Maroantsetra, Makira, 15²18'11"S, 49³3'13.9"E, alt. 160 m, 20 May 2007, Rakotonasolo et al. 1117 (K, TAN); Maroantsetra, summit of Ankirindro massif, $15^{\circ} 18^{\prime} 08^{\prime \prime} \mathrm{S}, 4^{\circ} 33^{\prime} 04^{\prime \prime E}$, alt. $671 \mathrm{~m}, 1$ Feb. 1999, Schatz 3916 (P, MO).

HABITAT. Open mid-elevation humid forests, altitude 640 - $981 \mathrm{~m}$.

CONSERVATION STATUS. This species has an AOO of 12 $\mathrm{km}^{2}$, and an EOO of $2.15 \mathrm{~km}^{2}$. The two locations known are outside of protected areas, and the species is threatened by habitat loss due to forest logging and shifting agriculture. This species is rated as Endangered (EN B2ab(i,ii,iii)).

ETYMOLOGY. The species epithet is derived from the name of the place where the type was collected.

2. Phyllanthus humbertii (Leandri) Petra Hoffm. E McPherson (2003: 308). Glochidion humbertii Leandri (1957: 215). Type: Madagascar, Massif de Marojejy, à l'Ouest de la rivière Manantenina, forêt humide sur laterite et gneiss, alt. $1450 \mathrm{~m}$, Dec. 1948, Humbert 22495 (holotype P!, isotype WAG).
Monoecious shrubs or sometimes sarmentose shrubs. Branching pinnatiform; orthotropic branches flattened, striate, glabrous. Cataphylls triangular, $1-1.3$ $\times 0.6-1 \mathrm{~mm}$, coriaceous, entire, glabrous. Cataphyllary stipules triangular, $1-1 \times 0.6-1 \mathrm{~mm}$, coriaceous, entire, glabrous. Plagiotropic branches solitary, compressed, $10-18 \mathrm{~cm}$ long, $1-1.5 \mathrm{~mm}$ diam., striate, glabrous. Stipules caducous or persistent, triangular, $0.4-0.7 \times 0.2-0.3 \mathrm{~mm}$, membranous, entire, glabrous. Leaves $7-11$ per branch. Internodes $5-20.5 \mathrm{~mm}$ long. Petiole terete, $4-6 \times$ 0.7 - $1 \mathrm{~mm}$, glabrous. Leaf blades ovate, $25-80 \times 14-$ $45 \mathrm{~mm}, 1.8 \times$ longer than wide, rounded to truncate at the base, acuminate at the apex, acumen $1-2 \mathrm{~cm}$, chartaceous, glabrous on both sides; midvein prominent on both sides; secondary veins $2-4$ pairs, prominent on both sides. Inflorescence bisexual, consisting of 1 - 4 male flowers and one female flower, or solitary female flower. Bracts triangular, 0.8 $-1 \times 0.4-0.5 \mathrm{~mm}$, entire or slightly erose, glabrous. Male flowers $2.7-4.3 \times 1.8-2.6 \mathrm{~mm}$. Pedicels $2-4 \times$ $0.3-0.4 \mathrm{~mm}$, glabrous. Tepals 5, unequal, oblong, $3-$ $4 \times 1.3-1.5 \mathrm{~mm}$, round or obtuse, coriaceous, glabrous on both sides, margin entire, hyaline for $1 / 5$ of entire tepal width on each side, veins unbranched. Disc glands 5, globose, c. $0.2 \mathrm{~mm}$ in diam. Stamens 3, entirely fused into a column; column terete, enlarged at the base, $1.3-3 \times 0.6-0.8 \mathrm{~mm}$; anthers fused, oblong, $0.7-1 \times 0.3 \mathrm{~mm}$, not apiculate. Female flowers $1.5-2 \times 1-1.5 \mathrm{~mm}$. Pedicels $18-25 \times 0.4-$ $0.5 \mathrm{~mm}$, glabrous. Tepals 5 , equal, ovate, $1-2 \times$ c. 0.5 $\mathrm{mm}$, obtuse, subcoriaceous, margin slightly erose, hyaline for $1 / 5$ of entire tepal width, glabrous on both sides, veins unbranched. Disc extremely reduced or absent. Ovary 3-locular, ovoid, $2-3 \times 1.5-2 \mathrm{~mm}$, smooth, glabrous. Styles free, deltoid, entire, $0.4-$ $0.5 \mathrm{~mm}$ long; stigmas obtuse. Fruits globose, $4-5 \times 4-$ $5 \mathrm{~mm}$, glabrous; fruiting pedicels $20-27 \times 0.5-0.7$ $\mathrm{mm}$, glabrous; tepals persistent, $1-2 \times 0.5-1 \mathrm{~mm}$; columella $2-2.8 \times 0.5-0.6 \mathrm{~mm}$. Seeds $2.5-3 \times 1.5-2$ $\mathrm{mm}$, smooth.

DISTRIBUTION. Endemic to Madagascar. Central floristic domain. Map 2.

SPECIMENS EXAMINED. MADAGASCAR. Antsiranana: Massif de Marojejy, à l'Ouest de la rivière Manantenina, forêt humide sur laterite et gneiss, alt. 1450 m, Dec. 1948, Humbert 22495 (P).

HABITAT. Open humid forests, altitude $1450 \mathrm{~m}$.

CONSERVATION STATUS. Phyllanthus humbertii is known from two collections: the type and another specimen from Marojejy made by Kawakita $\mathcal{E}^{2}$ Kato 235 (KYO not seen). This species is probably very rare and its area of occupancy (AOO) is estimated to be less than $10 \mathrm{~km}^{2}$. The species is threatened by 


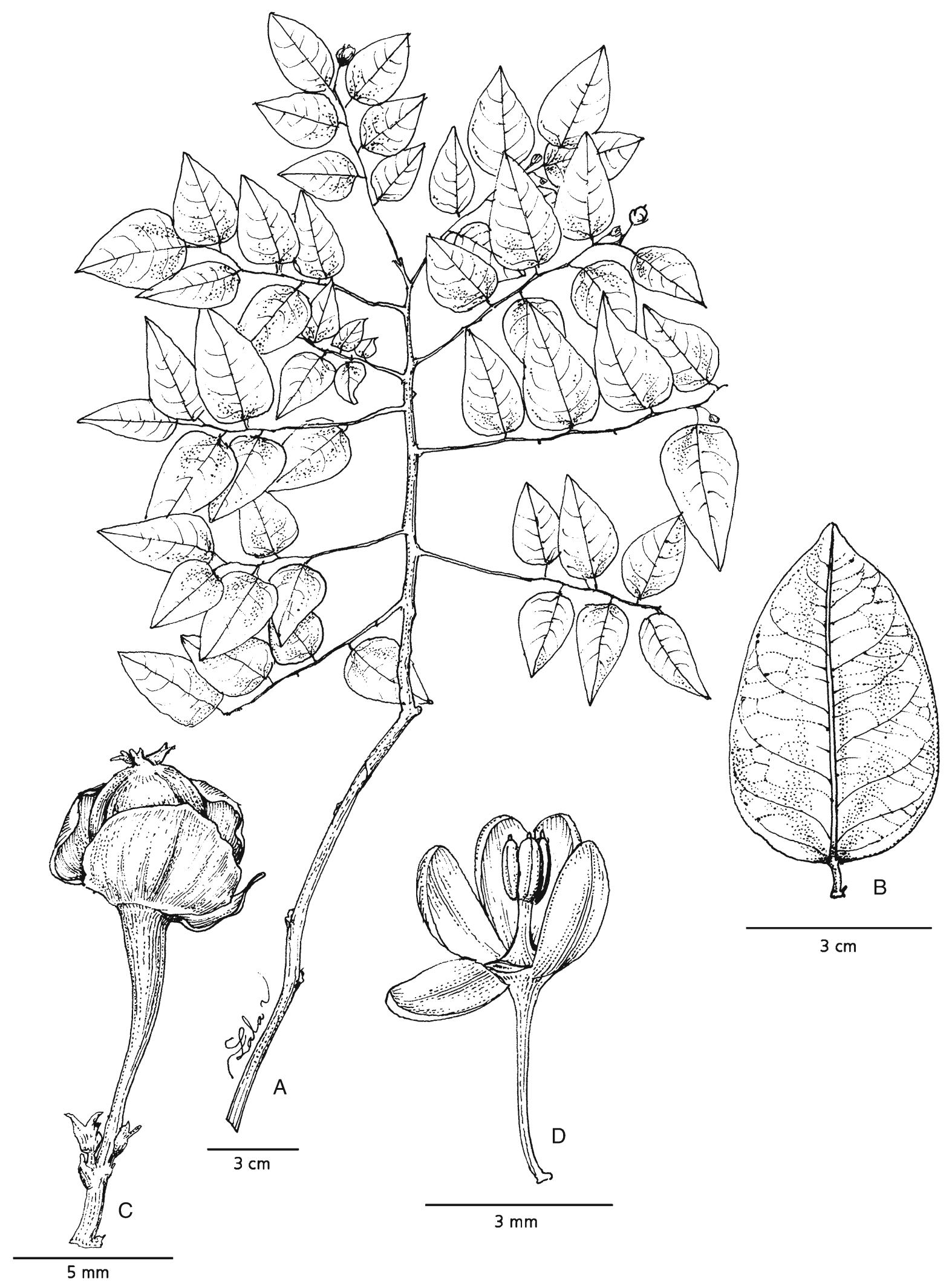

Fig. 1. Phyllanthus ankirindrensis (G. E. Schatz 3916, P). A habit; B leaf; C female flower; D male flower. DRAWN BY R. L. ANDRIAMIARISOA. 


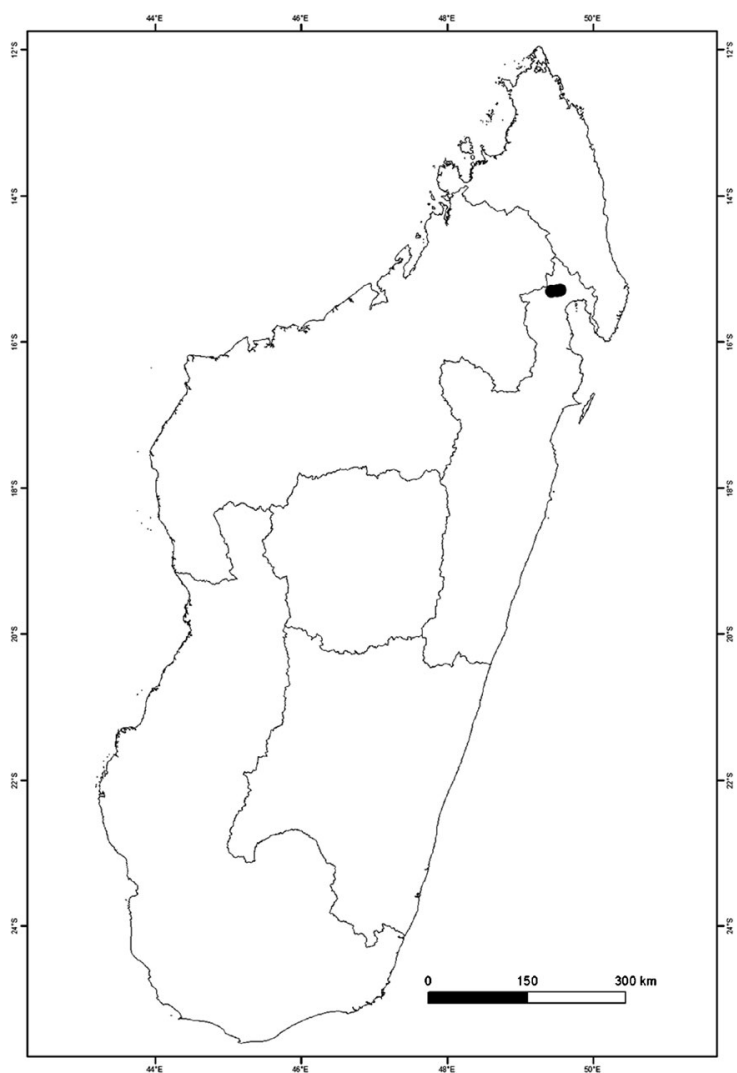

Map 1. Distribution of Phyllanthus ankirindrensis in Madagascar.

habitat loss due to illegal logging. It is therefore rated as Critically Endangered (CR B2ab(ii,iii,iv)).

3. Phyllanthus razakamalalae Ralim. E Gable, sp. nov. Type: Madagascar. Antsiranana: Antomboka, Ambodimanga, Ambanja, 1345'12"S, 048 31'19"E, alt. $41 \mathrm{~m}, 4$ Dec. 2000, Razakamalala et al. 45bis (holotype $\mathrm{K}$ !; isotype $\mathrm{MO}$ ).

http:/ /www.ipni.org/urn:lsid:ipni.org:names:77213111-1

Monoecious shrubs, $0.5-1 \mathrm{~m}$ high. Branching pinnatiform; orthotropic branches terete, smooth or striate, glabrous. Cataphylls triangular, $0.4-0.7 \times 0.3$ - $0.4 \mathrm{~mm}$, coriaceous, entire, glabrous. Cataphyllary stipules triangular, $0.3-0.5 \times 0.3-0.4 \mathrm{~mm}$, coriaceous, entire, glabrous. Plagiotropic branches solitary, terete, $15-24 \mathrm{~cm}$ long, $0.5-0.8 \mathrm{~mm}$ in diam., striate, glabrous or with thin hairs. Stipules persistent, triangular, $1-1.2 \times 0.2-0.3 \mathrm{~mm}$, membranous or chartaceous, entire, glabrous. Leaves 9 - 20 per branch. Internode $6-10 \mathrm{~mm}$ long. Petioles terete, 1 $-2 \times 0.2-0.3 \mathrm{~mm}$, glabrous. Leaf blades obovate to oblong, $12-40 \times 7-11 \mathrm{~mm}, 1.7-3.6 \times$ longer than wide, obtuse at the base, rounded sometimes with short apiculum $1-1.5 \mathrm{~mm}$ long at the apex,

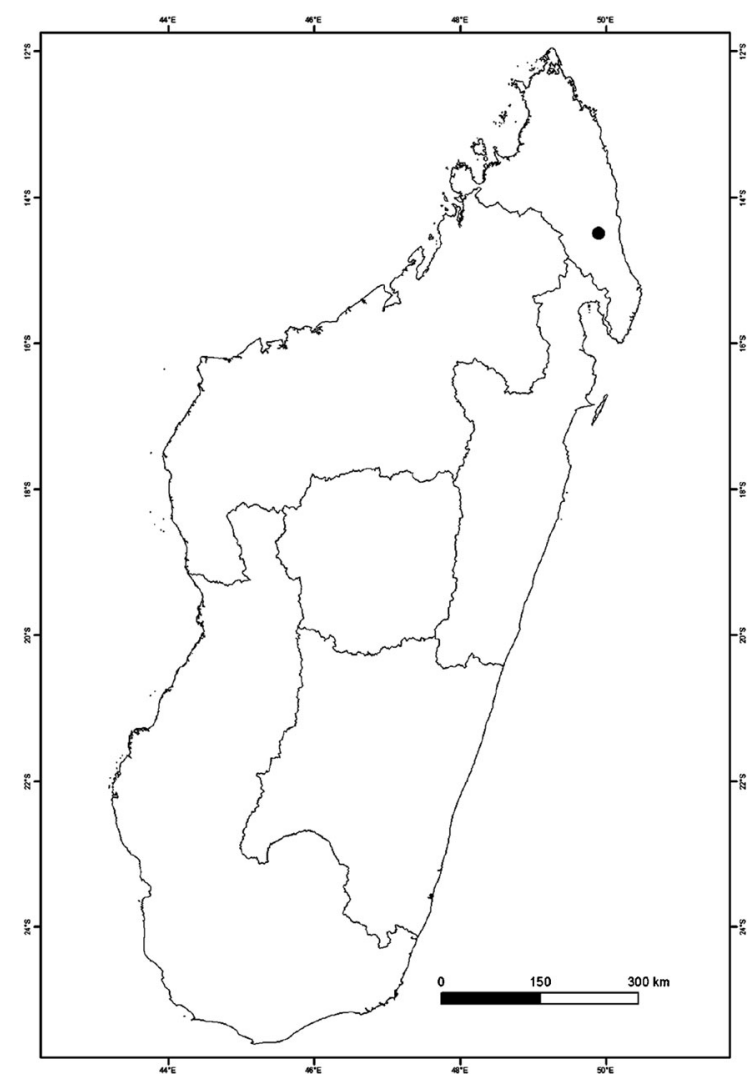

Map 2. Distribution of Phyllanthus humbertii in Madagascar.

membranous or chartaceous, glabrous on both sides; midvein flattened adaxially, prominent abaxially; secondary veins $3-5$ pairs, flattened on both sides. Inflorescences bisexual or unisexual, consisting of $1-3$ male flowers and one female flower or solitary female flower. Bracts triangular, $0.3-0.4 \times 0.2-0.3 \mathrm{~mm}$, entire, glabrous. Male flowers $2-2.5 \times 1.8-2.5 \mathrm{~mm}$. Pedicels $1-2 \times 0.3 \mathrm{~mm}$, glabrous. Tepals 5 , unequal, oblong to elliptic, $1.5-2 \times 0.5-0.8 \mathrm{~mm}$, rounded or obtuse, coriaceous, glabrous on both sides, margin entire, hyaline for $1 /{ }_{6}$ of total tepal width on each side, veins unbranched. Disc glands 5 , globose, c. $0.2 \times 0.2$ $\mathrm{mm}$. Stamens 5, entirely fused in column; column terete, $0.9-1.2 \times 0.4 \mathrm{~mm}$; anthers fused, oblong, not apiculate, c. $0.8 \times 0.3 \mathrm{~mm}$. Female flowers $1-1.3 \times 0.5$ $-1 \mathrm{~mm}$. Pedicels c. $1 \times 0.3 \mathrm{~mm}$, glabrous. Tepals 5 , subequal, oblong to elliptic, $0.7-1.3 \times 0.4-0.5 \mathrm{~mm}$, obtuse, coriaceous, glabrous on both sides, margin entire, hyaline for $1 / 5$ of entire tepal width on each side, veins unbranched. Disc lobed. Ovary 3-locular, ovoid or subglobose, $0.8-1 \times 0.8-1 \mathrm{~mm}$, smooth, glabrous. Styles free, deltoid, entire or slightly bifid at the apex, $0.5-0.7 \mathrm{~mm}$ long, stigmas obtuse. Fruits ovoid or subglobose, c. $2 \times 1.8 \mathrm{~mm}$, glabrous; fruiting pedicels c. $3 \times 0.5 \mathrm{~mm}$, glabrous; tepals $0.9-1.2 \times 0.4$ $-0.5 \mathrm{~mm}$; columella $1-1.3 \times 0.3 \mathrm{~mm}$. Seeds $1-1.2 \times$ $0.7-1 \mathrm{~mm}$, thinly striated longitudinally. Fig. 2. 


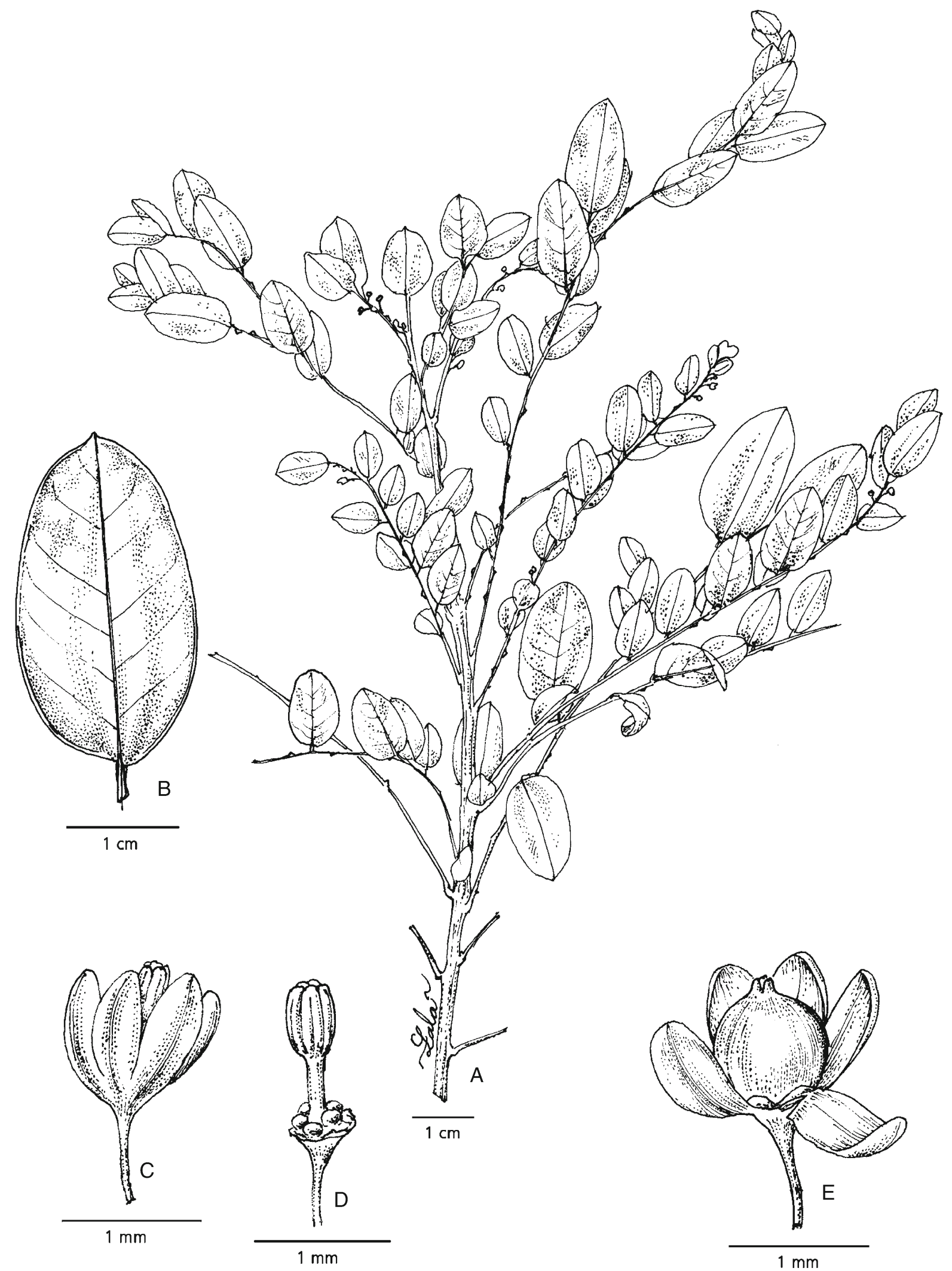

Fig. 2. Phyllanthus razakamalalae (C. Birkinshaw 1047, TAN). A habit; B leaf; C male flower; D stamens; E female flower. DRAWN BY R. L. ANDRIAMIARISOA. 
RECOGNITION. Phyllanthus razakamalalae is similar to $P$. humbertii by the bracts not in a cluster, the filaments and anthers entirely fused, not apiculate, and the deltoid free styles. It differs from $P$. humbertii in the leaf blades obovate to oblong (vs ovate), obtuse at the base (vs rounded to truncate), rounded at the apex sometimes with a short apiculum (vs acuminate, acumen $1-2 \mathrm{~cm}$ long) and the 5 stamens (vs 3).

DISTRIBUTION. Endemic to Madagascar. Central floristic domain. Map 3.

PARATYPE: MADAGASCAR. Antsiranana: Reserve Naturelle Intégrale Tsaratanana, R. Ramena, 1 $2 \mathrm{~km}$ SW of junction with R. Andilambe, $13^{\circ} 52^{\prime} 20^{\prime \prime S}$, $048^{\circ} 50^{\prime} 50^{\prime \prime E}$, alt. 547 m, 14 Nov. 2001, Birkinshaw et al. 1047 (MO, TAN).

SPECIMENS EXAMINED. MADAGASCAR. Antsiranana: Réserve spéciale de Manongarivo, cours supérieur, $13^{\circ} 59^{\prime} \mathrm{S}, 48^{\circ} 26^{\prime} \mathrm{E}$, alt. $700 \mathrm{~m}, 6$ March 1999, Gautier et al. 3499 (G, K); Andapa, Doany Andranomololo, 14²1'34'S, 49²2'35"E, Rakotovao et al. 3199 (MO, TAN); Antomboka, Ambodimanga, Ambanja, $13^{\circ} 45^{\prime} 12^{\prime \prime S}, 048^{\circ} 31^{\prime} 19^{\prime \prime E}$, alt. 41 m, 4 Dec. 2000, Razakamalala et al. 45bis (K, MO).

HABITAT. Margins of humid forest, altitude $700 \mathrm{~m}$. CONSERVATION STATUS. Phyllanthus razakamalalae has an AOO of $16 \mathrm{~km}^{2}$ and an EOO of $2081.45 \mathrm{~km}^{2}$. It is

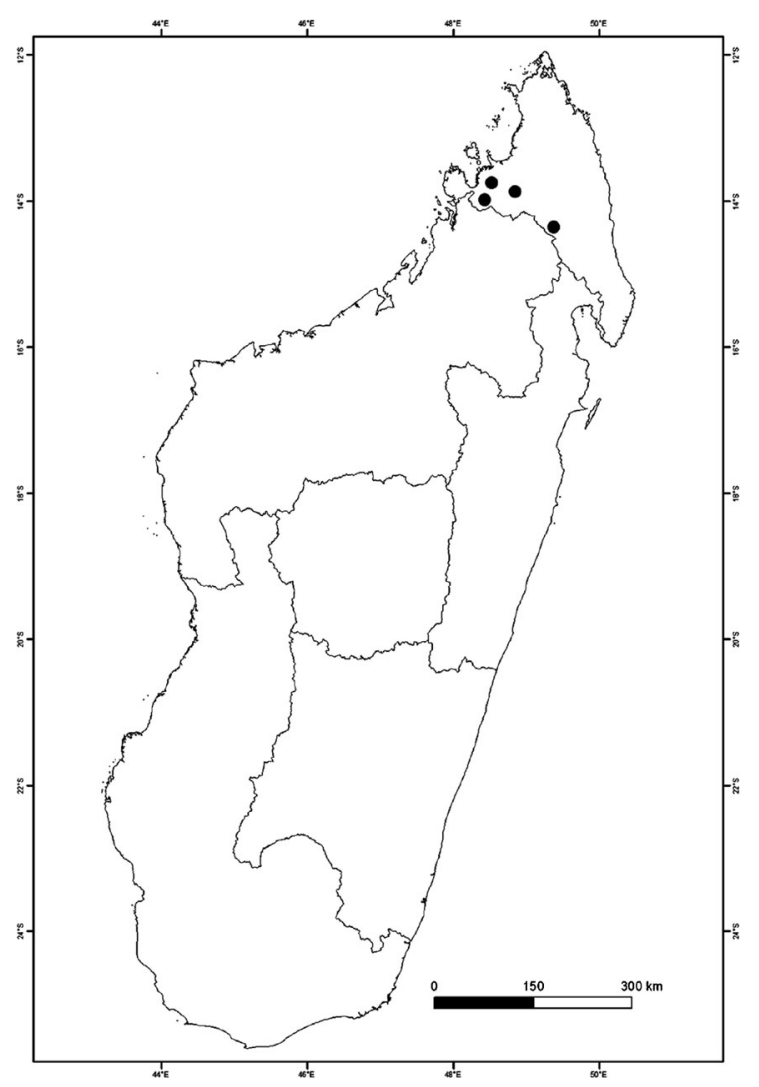

Map 3. Distribution of Phyllanthus razakamalalae in Madagascar. known from four locations, two subpopulations are inside protected areas (Manongarivo and Tsaratanana). The subpopulations outside of the protected areas are threatened by habitat loss due to shifting agriculture, forest logging and annual fire. It is assessed as Endangered (EN Blab(i,ii,iii,iv) + 2ab(i,ii,iii,iv)).

ETYMOLOGY. The species epithet honours our colleague R. Razakamalala who collected the type specimen.

4. Phyllanthus oreichtitus Leandri (1935: 24). Phyllanthus monticola Leandri (1934: 450), nom. illegit. Glochidion oreichtitum (Leandri) Leandri (1937: 29). Type: Madagascar, Centre: Andringitra, forêt à mousses et sous-bois herbacé, at. 1800 - 2000 m, April 1921, Perrier de la Bâthie 13670 (lectotype P!, selected by Brunel (1987)).

Glochidion oreichtitum subsp. tsaratanense Leandri (1957: 217). Type: Madagascar, Centre-nord, mont Tsaratanana, alt. 2000 m, Oct. 1919, Perrier de la Bâthie 9714 (holotype P!).

Monoecious shrubs, 2 - $3 \mathrm{~m}$ high. Branching pinnatiform; orthotropic branches terete, $2.5-3 \mathrm{~mm}$ in diam., rugose or striate. Cataphylls triangular, $1.2-1.5 \times$ $0.5-0.9 \mathrm{~mm}$, coriaceous, entire, glabrous. Cataphyllary stipules triangular, c. $1 \times 0.7 \mathrm{~mm}$, coriaceous, entire, glabrous. Plagiotropic branches solitary, terete or flattened toward the apex, $4-15 \mathrm{~cm}$ long, $1-1.4 \mathrm{~mm}$ in diam., pubescent. Stipules persistent, triangular, $1.8-2.5$ $\times 0.5-0.8 \mathrm{~mm}$, coriaceous, entire, glabrous. Leaves $8-22$ per branch. Internodes 3-7 mm long. Petioles terete, c. $1 \times 0.4-0.8 \mathrm{~mm}$, glabrous. Leaf blades ovate, $4-40 \times 7-$ $16 \mathrm{~mm}, 0.5-2.5 \times$ longer than wide, obtuse at the base, attenuate to acuminate at the apex, chartaceous, glabrous on both sides; midvein prominent on both sides; secondary veins $3-5$ pairs, prominent on both sides. Inflorescences unisexual, solitary, male inflorescences and female inflorescences on different branches; bracts triangular or ovate, $0.5-0.8 \times 0.2-0.3 \mathrm{~mm}$, entire, glabrous. Male flowers $2.5-3 \times 2-2.5 \mathrm{~mm}$. Pedicels $3-7$ $\times 0.3-0.4 \mathrm{~mm}$, glabrous. Tepals 6 , unequal, elliptic or oblong, $1.5-3 \times 0.7-1 \mathrm{~mm}$, round or obtuse, coriaceous, glabrous on both sides, margin entire, hyaline for $1 / 5$ of tepals' width on each side, veins unbranched. Disc glands 6 , globose or lenticular, $0.2-0.4 \mathrm{~mm}$ in diam. Stamens 6 in two whorls, the outer short free, the inner longer fused up to $1 / 3$ their length, filaments terete or subangular, $0.8-$ $2 \times 0.4-0.7 \mathrm{~mm}$; anthers free, oblong, apiculate, $0.6-0.9$ $\times 0.2-0.3 \mathrm{~mm}$. Female flowers $2.5-3 \times 2.5-3 \mathrm{~mm}$. Pedicels $7-20 \times 0.4-0.5 \mathrm{~mm}$, glabrous. Tepals 6 , unequal, obovate or suborbicular, $2-3 \times 1-1.5 \mathrm{~mm}$, rounded, coriaceous, glabrous on both sides, margin entire, hyaline for $1 / 4$ of tepal width on each side, veins 
unbranched. Disc lobed. Ovary 3-locular, globose, 2 - 2.6 $\times 1.8-2.6 \mathrm{~mm}$, smooth or slightly rugose, glabrous. Styles 3 , free, deltoid, entire or slightly bifid, $0.5-0.7 \mathrm{~mm}$ long; stigmas obtuse. Fruits depressed globose, $3-4 \times 3.5-4.5$ mm, glabrous; fruiting pedicels $7-20 \times 0.4-0.5 \mathrm{~mm}$; tepals persistent, $1.3-1.8 \times 1.2-1.5 \mathrm{~mm}$; columella c. $1.4 \mathrm{~mm} \times 0.5 \mathrm{~mm}$. Seeds c. $2 \times 2.4 \mathrm{~mm}$, smooth to thinly striated longitudinally. Fig. 3 .

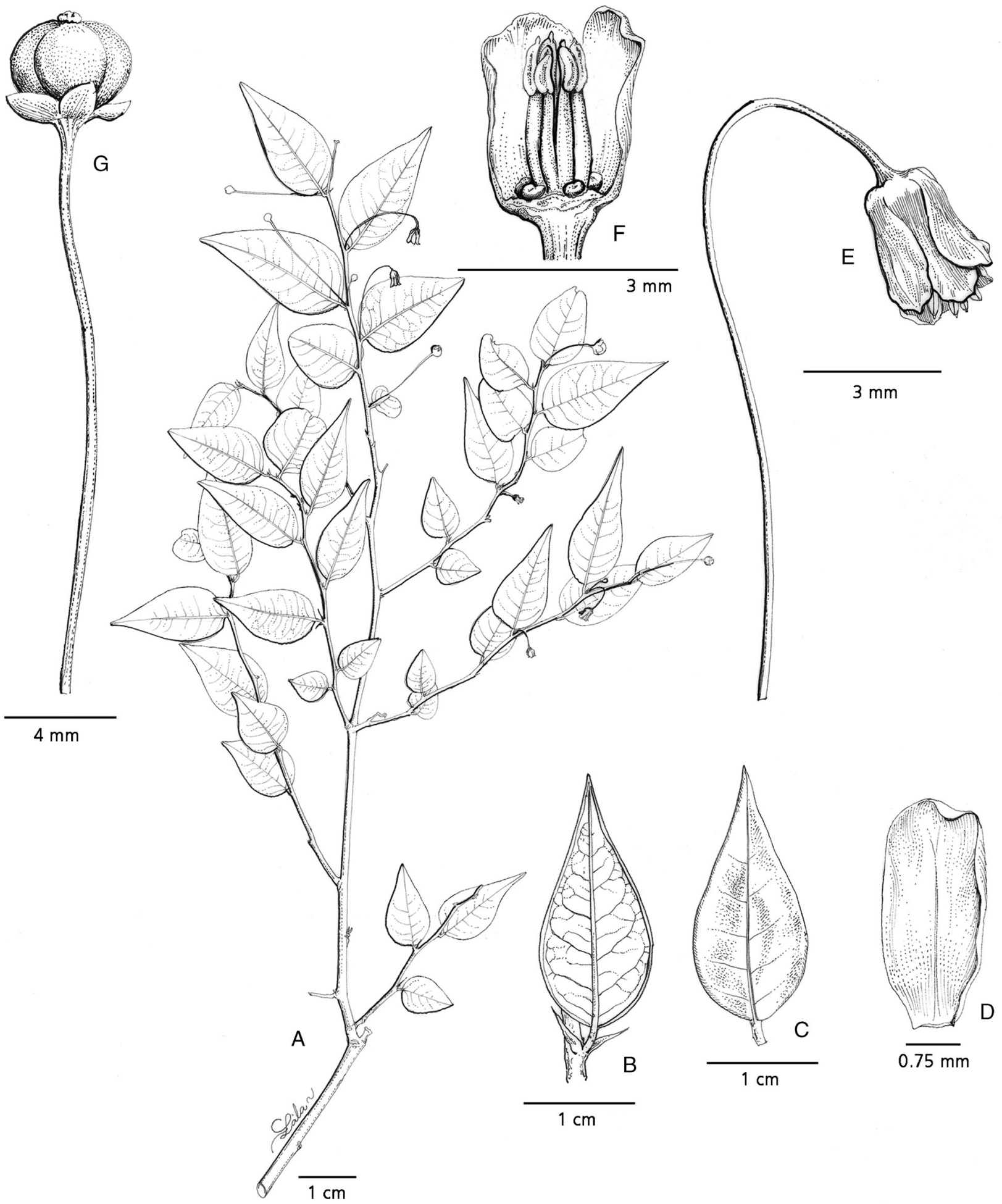

Fig. 3. Phyllanthus oreichtitus (J. Miller \& P. B. Phillipson 3717, TAN). A habit; B leaf (abaxial surface); C leaf (adaxial surface); D tepal; $E$ male flower; $F$ stamens; $G$ fruit. DRAWN BY R. L. ANDRIAMIARISOA. 
DISTRIBUTION. Endemic to Madagascar. Central floristic domain. Map 4.

SPECIMENS EXAMINED. MADAGASCAR. Antananarivo: Centre-Est de Marovoay N of Moramanga, 1 Sept. 1958, Capuron 18783 (P); Ankazobe, $18^{\circ} 15$ 'S, $47^{\circ} 9^{\prime} \mathrm{E}$, alt. 850 m, 29 Nov. 1988, Miller $\mathcal{E}$ Phillipson 3717 (K, P). Antsiranana: Massif of Tsaratanana, alt. $2100 \mathrm{~m}$, Nov. 1966, Morat 2258 (P); Ambanja, Marotaolana, massif of Tsaratanana, $14^{\circ} 09^{\prime} 19^{\prime \prime} \mathrm{S}, 48^{\circ} 57^{\prime} 28^{\prime \prime} \mathrm{E}$, alt. 2063 m, 24 April 2001, Rabenantoandro et al. 506 (K, MO, TAN); Tsaratanana, Bealanana, Mangidrano, Montagne d'Ampitsinjovana, est campement lac Matsaborimaika, $14^{\circ} 08^{\prime} 53^{\prime \prime} \mathrm{S}, 48^{\circ} 57^{\prime} 57^{\prime \prime} \mathrm{E}$, alt. $2275 \mathrm{~m}$, 17 April 2003, Reza et al. 322 (MO, TAN). Fianarantsoa: Ialatsara, Ambohimahasoa, 7 Feb. 1942, Decary 17474 (P); Ialatsara, Ambohimahasoa, 7 Feb. 1942, R. Decary 17483 (P); Andringitra, forêt à mousses et sous-bois herbacé, at. 1800 - 2000 m, April 1921, Perrier de la Bâthie 13670 (P); Andringitra massif, alt. $1800 \mathrm{~m}$, March 1922, Perrier de la Bâthie 14406 (P); Andranomainty, Ambalamanakana, 25 Feb. 1993, Rabevohitra et al. 2968R631 (TEF). Toamasina: Parc national Zahamena, Ihofika, South-East Manakambahiny, $17^{\circ} 39^{\prime} 13^{\prime \prime S}, 48^{\circ} 57^{\prime} 50^{\prime \prime E}$, alt. $550 \mathrm{~m}$, 22 Oct. 2002, Randrianasolo 328 (K, MO).

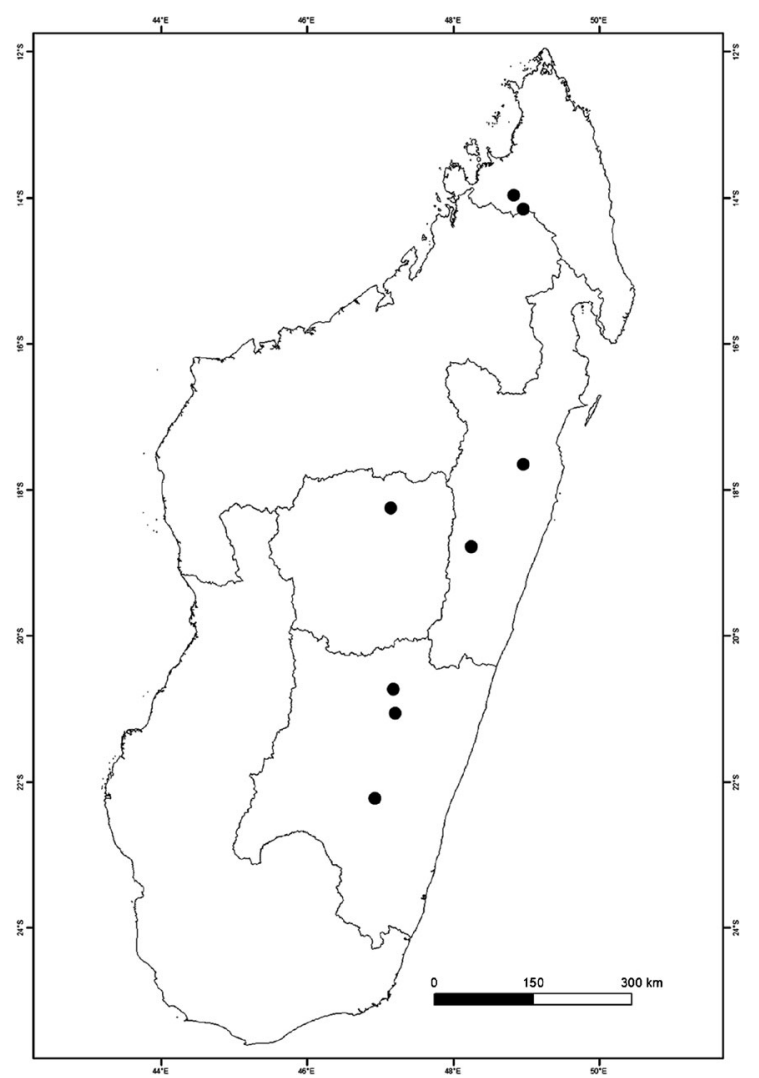

Map 4. Distribution of Phyllanthus oreichtitus in Madagascar.
HABITAT. Margin of humid forests, open humid forests. Altitude $850-2000 \mathrm{~m}$.

CONSERVATION STATUS. Phyllanthus oreichtitus has an AOO of $32 \mathrm{~km}^{2}$, and an EOO of $84305.72 \mathrm{~km}^{2}$. Two subpopulations are found in the protected areas of Andringitra and Tsaratanana. This species is rated as Least Concern (LC).

NOTES. In his description Leandri (1957) recognised Phyllanthus oreichtitus subsp. tsaratanensis. He distinguished it from $P$. oreichtitus subsp. oreichtitus only by the leaf size and the fruiting pedicel length (smaller and shorter in $P$. oreichtitus subsp. tsaratanensis). We found no clear characters that distinguish between the two subspecies as well as a general overlap in all morphological characters. In our opinion there is no reason to differentiate two subspecies. In addition, $P$. oreichtitus has a large distribution area in Madagascar and is recorded in different types of habitat. The leaf size reduction is among of the usual adaptation characters in high altitude. In this treatment we put Phyllanthus oreichtitus subsp. tsaratanensis into synonymy.

5. Phyllanthus perrieri (Leandri) Petra Hoffm. E McPherson (2003: 308). Glochidion perrieri Leandri (1934: 606). Type: Madagascar, Massif of Manongarivo, alt. 800 - 1000 m, May 1909, Humbert 9918 (holotype P!).

Monoecious shrubs, 1 - $2 \mathrm{~m}$ high. Branching pinnatiform; orthotropic branches terete or angular toward the apex, rugose or striate, glabrous. Cataphylls triangular, $1.2-1.5 \times 0.5-0.6 \mathrm{~mm}$, coriaceous, entire, glabrous. Cataphyllary stipules triangular, $1-1.3 \times 0.5$ - $0.6 \mathrm{~mm}$, coriaceous, entire, glabrous. Plagiotropic branches solitary, terete or becoming angular toward the apex, $7-20 \mathrm{~cm}$ long, $1-1.5 \mathrm{~mm}$ in diam., striate, glabrous. Stipules persistent or caducous, triangular, $0.6-1.3 \times 0.4-0.6 \mathrm{~mm}$, coriaceous, entire, glabrous. Leaves 9 - 13 per branch. Internodes 3-12 mm long. Petioles flattened, $1-1.5 \times 0.8-1 \mathrm{~mm}$, glabrous. Leaf blades ovate to elliptic, symmetrical, $9-45 \times 7.5-22$ $\mathrm{mm}, 1.2-1.8 \times$ longer than wide, attenuate at the base, acute at the apex, flat, sub-coriaceous, glabrous on both sides; midvein flattened adaxially, prominent abaxially; secondary veins $3-5$ pairs, branched at the margin, flattened adaxially, prominent abaxially. Inflorescences unisexual consisting of $1-2$ male flowers or solitary female flower; male inflorescences and female inflorescences on different branches. Bracts triangular or obovate, in a cluster of 3-6 mainly sterile bracts in male inflorescence, $0.6-1 \times 0.4-0.5 \mathrm{~mm}$, entire or slightly erose, glabrous. Male flowers $3-4 \times 2-3 \mathrm{~mm}$. Pedicels $4-6 \times 0.3-0.4 \mathrm{~mm}$, glabrous. Tepals 5, unequal, ovate or oblong, $3-4 \times 1.5-2 \mathrm{~mm}$, round or 
obtuse, coriaceous, glabrous on both sides, margin entire, hyaline for $1 / 5$ of entire tepal width on each side, veins branched. Disc glands 5, lenticular, c. $0.2 \mathrm{~mm}$ in diam. Stamens 5 entirely free; anthers free, oblong, apiculate, $0.5-0.7 \times 0.2-0.3 \mathrm{~mm}$. Female flowers $4-5 \times 3-3.5 \mathrm{~mm}$. Pedicels $2-2.4 \times$ c. 0.5 $\mathrm{mm}$, glabrous. Tepals 5 , unequal, elliptic or obovate, 4 $-5 \times 2-5 \mathrm{~mm}$, obtuse, coriaceous, glabrous on both sides, margin entire, hyaline for $1 / 6$ of entire tepal width on each side, veins branched. Disc lobed. Ovary 3-locular, ovoid or globose, c. $2 \times 1.7-2 \mathrm{~mm}$, smooth, glabrous. Styles free, deltoid, entire, $1-1.5 \mathrm{~mm}$ long; stigmas obtuse. Fruits ovoid or globose, $3.5-4 \times 3-4$ $\mathrm{mm}$, glabrous; fruiting pedicels $2.4-3 \times 0.5-0.7 \mathrm{~mm}$, glabrous; tepals persistent $4.2-5 \times 2-5.3 \mathrm{~mm}$; columella $1-1.2 \times 0.5-0.6 \mathrm{~mm}$. Seeds c. $2.5 \times 1.7 \mathrm{~mm}$, finely striated longitudinally.

DISTRIBUTION. Endemic to Madagascar. Sambirano floristic domain. Map 5.

SPECIMENS EXAMINED. MADAGASCAR. Antsiranana: Tsaratanana, Antsaharatsy, Antsahabe, Mandrizavona, $13^{\circ} 47^{\prime} 56^{\prime \prime} \mathrm{S}, 48^{\circ} 48^{\prime} 20$ "E, alt. $1300 \mathrm{~m}, 16$ April 2000, Antilahimena et al. 480 (K, MO); Manongarivo, Bekolosy, $14^{\circ} 02 ' \mathrm{~S}, 48^{\circ} 18^{\prime} \mathrm{E}$, alt. 1100 m, 24 Aug. 1994,. Dereleth 117 (G, K, P); Manongarivo, Besinkara, Ambalafary, $14^{\circ} 04^{\prime} \mathrm{S}, 48^{\circ} 17^{\prime} \mathrm{E}$, alt. $300 \mathrm{~m}, 14$ July 1994, Gautier Eे

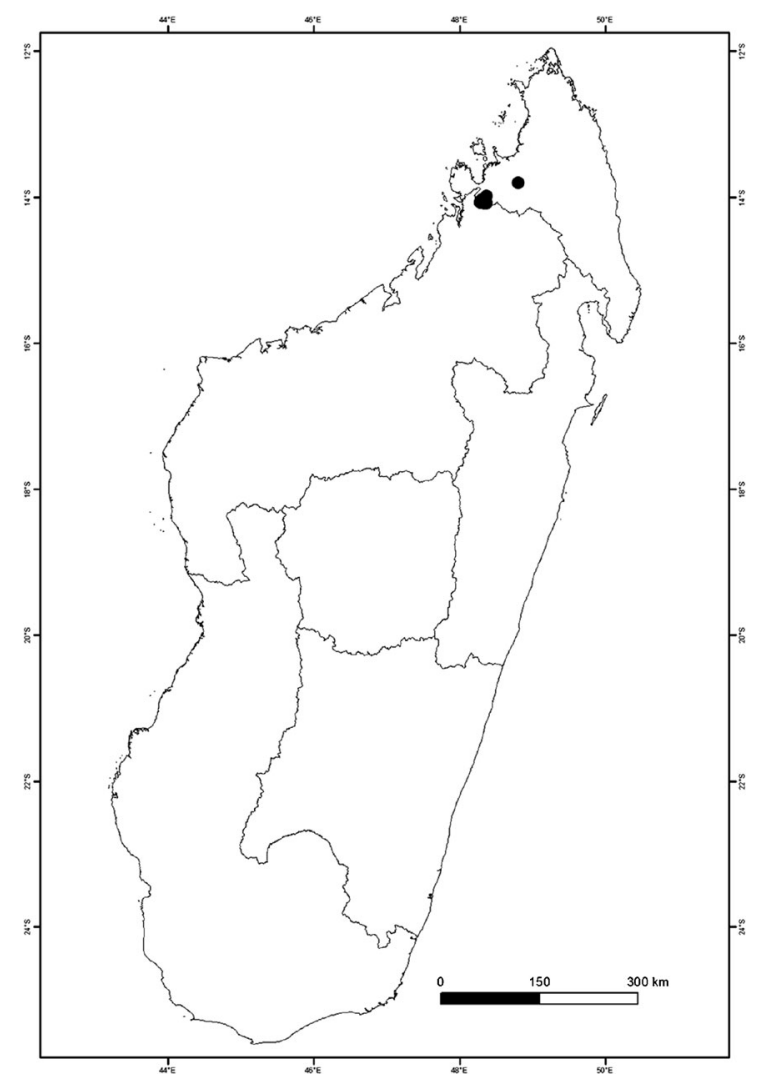

Map 5. Distribution of Phyllanthus perrieri in Madagascar.
Chatelain 2326 (G, K, P); Massif of Manongarivo, alt. 800 1000 m, May 1909, Humbert 9918 (P); Manongarivo, $14^{\circ} 05^{\prime} \mathrm{S}, 48^{\circ} 20^{\prime} \mathrm{E}$, alt. 1100 - $1200 \mathrm{~m}, 18$ Oct. 1994 , McPherson E Werff 16407 (MO); Manongarivo, Antsatrotro, $14^{\circ} 5^{\prime} \mathrm{S}, 48^{\circ} 22^{\prime} \mathrm{E}$, alt. $1280 \mathrm{~m}, 30$ Nov. 2000, Wohlhausser et al. 425 ( $\mathrm{G}, \mathrm{K})$.

HABITAT. Margins of humid forest, altitude $250-1300 \mathrm{~m}$. CONSERVATION StATUS. Phyllanthus perrieri has an AOO of $24 \mathrm{~km}^{2}$ and an EOO of $325.90 \mathrm{~km}^{2}$. Two subpopulations are found inside of Manongarivo and Tsaratanana protected areas. The subpopulations found outside of the protected area are threatened by shifting agriculture. This species is therefore rated as Endangered (EN Blab(i,ii,iii,iv) + 2ab(i,ii,iii,iv)).

6. Phyllanthus sambiranensis Leandri (1934: 451). Glochidion sambiranense (Leandri) (1937: 29). Type: Madagascar. Haut bassin de Sambirano, $500 \mathrm{~m}$, quartzite, Dec. 1912, Perrier de la Bâthie 9698 (lectotype P!, selected by Brunel (1987: 422)).

Glochidion sambiranense var. trapezophyllum Leandri (1958: 29), nom. inval.

Monoecious shrubs, $1-4 \mathrm{~m}$ high. Branching pinnatiform; orthotropic branches terete or angular toward the apex, striate, glabrous. Cataphylls triangular, $0.5-0.8 \times 0.5-0.6 \mathrm{~mm}$, coriaceous, entire, glabrous. Cataphyllary stipules triangular, $0.4-0.8 \times$ $0.5-0.6 \mathrm{~mm}$, coriaceous, entire, glabrous. Plagiotropic branches solitary, terete, $4.5-17 \mathrm{~cm}$ long, $0.5-$ $1 \mathrm{~mm}$ in diam., striate glabrous. Stipules persistent, triangular, $0.4-0.6 \times 0.3-0.4 \mathrm{~mm}$, coriaceous, entire, glabrous. Leaves 9 - 51 per branch. Internodes 1 $5 \mathrm{~mm}$ long. Petioles terete or flattened, $1-1.5 \times 0.5-$ $0.6 \mathrm{~mm}$, glabrous. Leaf blades asymmetrical elliptic or obovate, $6-13 \times 3-9 \mathrm{~mm}, 1.4-2 \times$ longer than wide, cuneate to obtuse at the base, acute at the apex, coriaceous, revolute, glabrous on both sides; midvein prominent on both sides; secondary veins $3-4$ pairs, prominent adaxially and abaxially. Inflorescences unisexual, solitary or fasciculate, consisting of $1-3$ male flowers ( 1 developed and the others still in bud) or solitary female flower, male and female inflorescences on different branches. Bracts elliptic or obovate, in a cluster of $3-6$ mainly sterile bracts in male inflorescences, $0.5-1 \times 0.3-0.5 \mathrm{~mm}$, entire, glabrous. Male flowers $2-2.5 \times 1.7-2 \mathrm{~mm}$. Pedicels $2-5 \times \mathrm{c}$. $0.4 \mathrm{~mm}$, glabrous. Tepals 5 , unequal (2 outer smaller), oblong or obovate, $1.5-2.2 \times 0.8-2 \mathrm{~mm}$, round, coriaceous, glabrous on both sides, margins entire, hyaline for $1 / 2$ of tepal width on each side, veins unbranched. Disc glands 5, globose, c. $0.2 \times 0.2 \mathrm{~mm}$. Stamens 5, entirely free; filaments angular, 0.6 - 1 $\times$ c. $0.4 \mathrm{~mm}$; anthers free, oblong, apiculate, $0.5-0.7 \times 0.2$ mm. Female flowers $2-2.5 \times 2-2.5 \mathrm{~mm}$. Pedicels 2.5 $-5 \times 0.3-0.5 \mathrm{~mm}$, glabrous. Tepals 5, subequal, 
oblong or ovate, $2-2.2 \times 1-1.2 \mathrm{~mm}$, obtuse, coriaceous, glabrous on both sides, margins entire, hyaline for $1 / 6$ of tepal width on each side, veins unbranched. Disc lobed. Ovary 3-locular, subglobose, $1.7-2 \times 1.7-2 \mathrm{~mm}$, smooth, glabrous. Styles entirely free, deltoid, entire, $0.4-0.6 \mathrm{~mm}$ long; stigmas obtuse. Fruits depressed globose, $2.8-3 \times$ c. $3.5 \mathrm{~mm}$, glabrous; fruiting pedicels $3-5 \times 0.3-0.4 \mathrm{~mm}$; tepals $2.2-2.8 \times$ $1-1.2 \mathrm{~mm} ; 1.3-1.6$; columella c. $1.5 \times 0.5 \mathrm{~mm}$. Seeds $1.8-2 \times$ c. $1.8 \mathrm{~mm}$, smooth. Fig. 4 .

DISTRIBUTION. Endemic to Madagascar. Eastern and Sambirano floristic domains. Map 6.

SPECIMENS EXAMINED. MADAGASCAR. Antsiranana: Sambava, Analamaho, forêt de Makirovana, 14²'52"S, 4956'33"E, alt. 842 m, 22 Aug. 2007, Andriamihajarivo et al. 1301 (MO, P, TAN); Réserve Spéciale de Manongarivo, Mont Antsatrotro, 1405'01"S, 048 23'18"E, alt. 1780 m, 14 Feb. 1994, Andrianarisata et al. 178 (MO); Ambatoharanana, Andranomena, Befandriana Nord, Makira, $15^{\circ} 07^{\prime} 58^{\prime \prime S}$, 49 $20^{\prime} 47^{\prime \prime E}$, alt. 1164 m, 14 Feb. 2008, Bernard 801 (MO, P, TAN); Diana, 13⒋ $5^{\prime} 31^{\prime \prime S}$, $048^{\circ} 05^{\prime} 27^{\prime \prime E}$, alt. 456 m, 1 March 2011, Bidault et al. 27 (G, MO, P, TAN); Manongarivo, Besinkara, below Ambalafary, Anabotoaka, $14^{\circ} 04^{\prime} \mathrm{S}, 48^{\circ} 17^{\prime} \mathrm{E}, 8$ April 1996, Gautier 3009 (G, TEF); East Ankaramy, summit ridge of Antsatrotro, Manongarivo, $14^{\circ} 05^{\prime} \mathrm{S}, 48^{\circ} 23^{\prime} \mathrm{E}$, alt. 17750 - $1850 \mathrm{~m}, 11$ April 1992, Malcomber et al. 1445 (MO, TAN); Sambava, Anjangoveratra, Anamboafo, Makirovana, $14^{\circ} 09^{\prime} 35^{\prime \prime} \mathrm{S}, 4^{\circ} 58^{\prime} 26^{\prime \prime E}$, alt. 642, 11 March 2014, Martial et al. 578 (MO, P, TAN); Vohemar, Antsirabe-Nord, Andravinambo, forêt d'Antsaolatra, $14^{\circ} 06^{\prime} 32^{\prime \prime S}, 49^{\circ} 57^{\prime} 34^{\prime \prime E}$, alt. $666 \mathrm{~m}, 11$ March 2014, Martial et al. 611 (MO, P, TAN); Manongarivo Massif, above village of Ambodisakoana, east of Ankaramy, $14^{\circ} 05^{\prime} \mathrm{S}, 48^{\circ} 20^{\prime} \mathrm{E}$, alt. $1110 \mathrm{~m}, 16$ Oct. 1994, McPherson, $\mathcal{E}$ Werff 16368 (MO, TAN); Manongarivo Massif, above village of Ambodisakoana, east of Ankaramy, $14^{\circ} 05^{\prime} \mathrm{S} 48^{\circ} 20^{\prime} \mathrm{E}$, alt. $1110-1200 \mathrm{~m}$, 18 Oct. 1994, McPherson E Werff 16401 (MO); Androranga, slope NE, alt. $1200 \mathrm{~m}$, rocky, quartzites, Perrier de la Bâthie 9728; Manongarivo massif, alt. 1000 $\mathrm{m}$, grès liasssiques, Perrier de la Bâthie 9717 (P); Haut bassin de Sambirano, 500 m, quartzite, Dec. 1912, Perrier de la Bâthie 9698 (P); Antsiranana, Massif Tsaratanana, alt. 100 m, Jan. 1923, Perrier de la Bathie 15488 (P); Massif Tsaratanana, alt. 2000 m, April 1924, Perrier de la Bathie 16414bis (P); Manongarivo, by Andranomavo, 12 Aug. 2004, Manongarivo, East of Antsatrotro, $48^{\circ} 23^{\prime} \mathrm{E}, 14^{\circ} 05^{\prime} \mathrm{S}$, alt. $1750-1850 \mathrm{~m}$, Malcomber et al. 1445 (MO); Belinta, Kalobinono, 1338'30"S, 48 39'48"E, alt. 492 m, 19 March 2009, Rajaovelona et al. 11 (K, TAN); Ambilobe, Manambato, Antsahavalany, $13^{\circ} 43^{\prime} 53^{\prime \prime S}, 49^{\circ} 23^{\prime} 38^{\prime \prime E}$, alt. 1455 m, 27 Oct. 2007, Randriambololomamonjy 157 (MO); Between
Maromandia and Ambanja, 1401'59.37"S, $48^{\circ} 09^{\prime} 14.46^{\prime E}$, alt. 127 m, 12 Sept. 2013, Ralimanana 1842 (K, TAN) ; Kalobinono, 13 38'23"S, 048 40'24"E, alt. 550 m, 28 Sept. 2013, Randriatsivery E Manjato 395 (G, MO, P, TAN); Galoko, 13 35'32"S, 048 42'36"E, alt. 125 m, 7 Oct. 2013, Randriatsivery et al. 450 (MO, P, TAN); Galoko, 1336'11"S, 4842' 37"E, alt. 430 m, 7 Oct. 2013, Randriatsivery et al. 477 (MO, P, TAN). Mahajanga: Analalava, Analantsoa, $14^{\circ} 2^{\prime} 44^{\prime \prime} \mathrm{S}$, $48^{\circ} 17^{\prime} 47^{\prime \prime E}$, alt. $1135 \mathrm{~m}, 12$ May 2014, Onjalalaina et al. 461 ( $\mathrm{K}, \mathrm{TAN})$.

HABITAT. Margins of humid forests, open humid forests. Altitude $120-2000 \mathrm{~m}$.

CONSERVATION STATUS. Phyllanthus sambiranensis has an EOO of $711268 \mathrm{~km}^{2}$, it has 10 locations. Three subpopulations are found in protected areas (Manongarivo, Tsaratanana). The subpopulations outside of protected areas are threatened by habitat degradation due to shifting agriculture, illegal forest exploitation. It is therefore assessed as Vulnerable (VU Blab(i, iii, iv)).

7. Phyllanthus marojejiensis (Leandri) Petra Hoffm. $\mathcal{E}$ McPherson (2003: 308). Glochidion marojejiense Leandri (1957: 215). Type: Madagascar. Sommet Est du massif de Marojejy, Ouest de Manantenina, affluent de Lokoho, alt. 1850 - 2137 m, Dec. 1948, Humbert 22761 (lectotype $\mathrm{P}$ !, selected here). This collection is chosen as lectotype because of its good preservation and its richness in flowers and fruits.

Monoecious shrubs or subshrubs. Branching pinnatiform; orthotropic branches terete, rugose or striated, glabrous. Cataphylls triangular, $0.7-1.5 \times 0.4$ - $0.5 \mathrm{~mm}$, coriaceous, entire, glabrous. Cataphyllary stipules triangular, $0.5-1.5 \times 0.3-0.5$, coriaceous, entire, glabrous. Plagiotropic branches solitary, terete, $1.5-5 \mathrm{~cm}$ long, $0.5-0.7 \mathrm{~mm}$ in diam., striate, glabrous. Stipules persistent, triangular or ovate, $0.8-$ $1.5 \times 0.3-0.5 \mathrm{~mm}$, coriaceous, entire, glabrous, hyaline margin white. Leaves 23 - 45 per branch. Internodes $0.5-1 \mathrm{~mm}$ long. Petioles absent. Leaf blades spatulate falcate, asymmetrical, concave abaxially, $4-6 \times 0.5-1.5 \mathrm{~mm}, 4.6-8 \times$ longer than wide, attenuate at the base, rounded at the apex, coriaceous, revolute, glabrous on both sides; midvein flattened adaxially, prominent abaxially; secondary veins indistinct on both sides. Inflorescences unisexual, solitary, male inflorescences on proximal and female inflorescences on distal part of plagiotropic branches or different branches. Bracts elliptic, in a cluster of 5 7 mainly sterile bracts in male inflorescences, $0.4 \times 0.3$ $\mathrm{mm}$, entire, glabrous. Male flowers $2-3.5 \times 1.5-2$ mm. Pedicels $0.5-1 \times 0.3-0.5 \mathrm{~mm}$, glabrous. Tepals 5, subequal, oblong, $2-3.5 \times 1-1.6 \mathrm{~mm}$, round, 


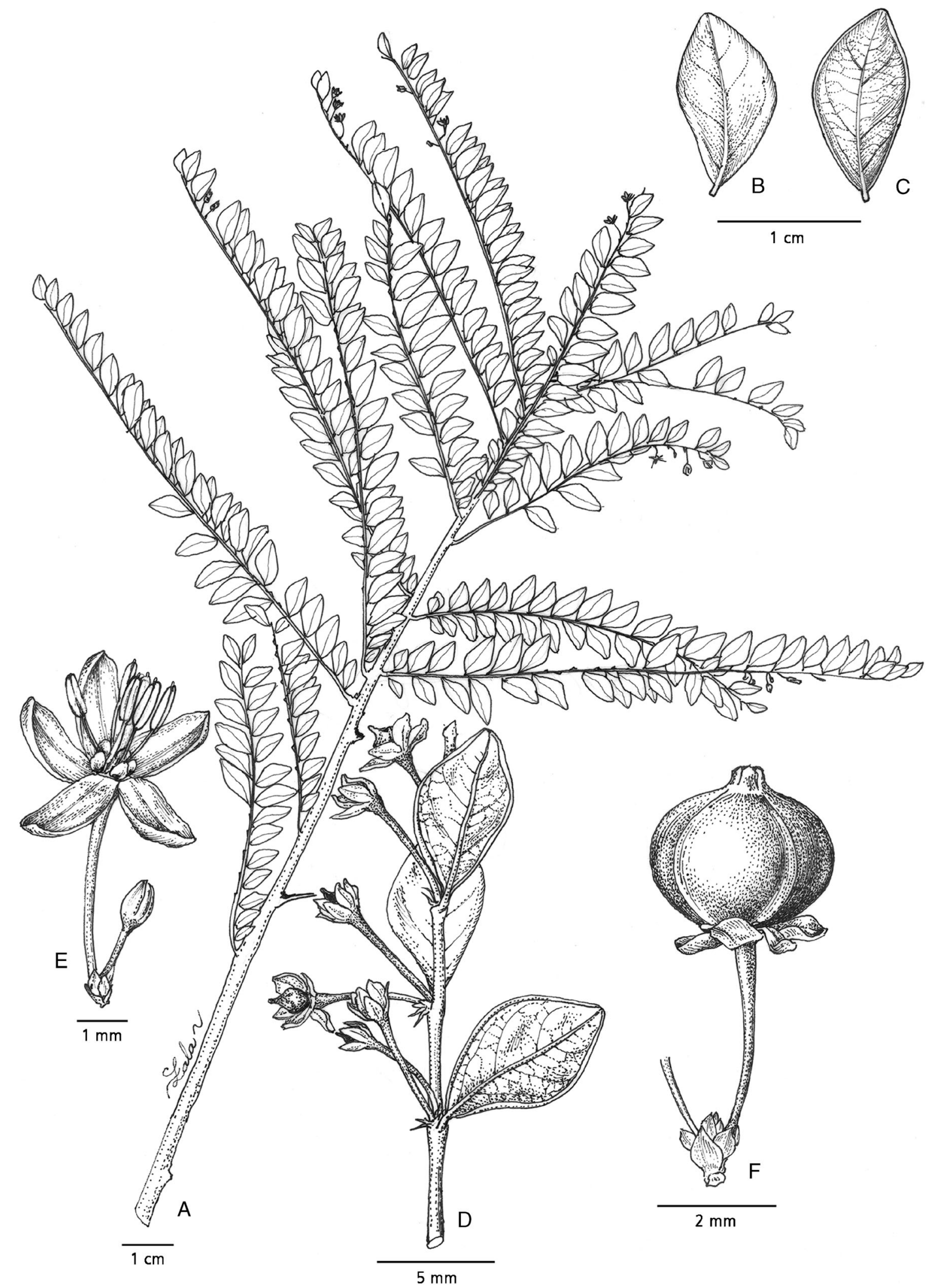

Fig. 4. Phyllanthus sambiranensis (S. Malcomber et al. 1445, G. McPherson \& H. van der Werff 16368, TAN). A habit; B leaf (adaxial surface); C leaf (abaxial surface); D branch with inflorescences; E male flower; F female flower. DRAWN BY R. L. ANDRIAMIARISOA. 


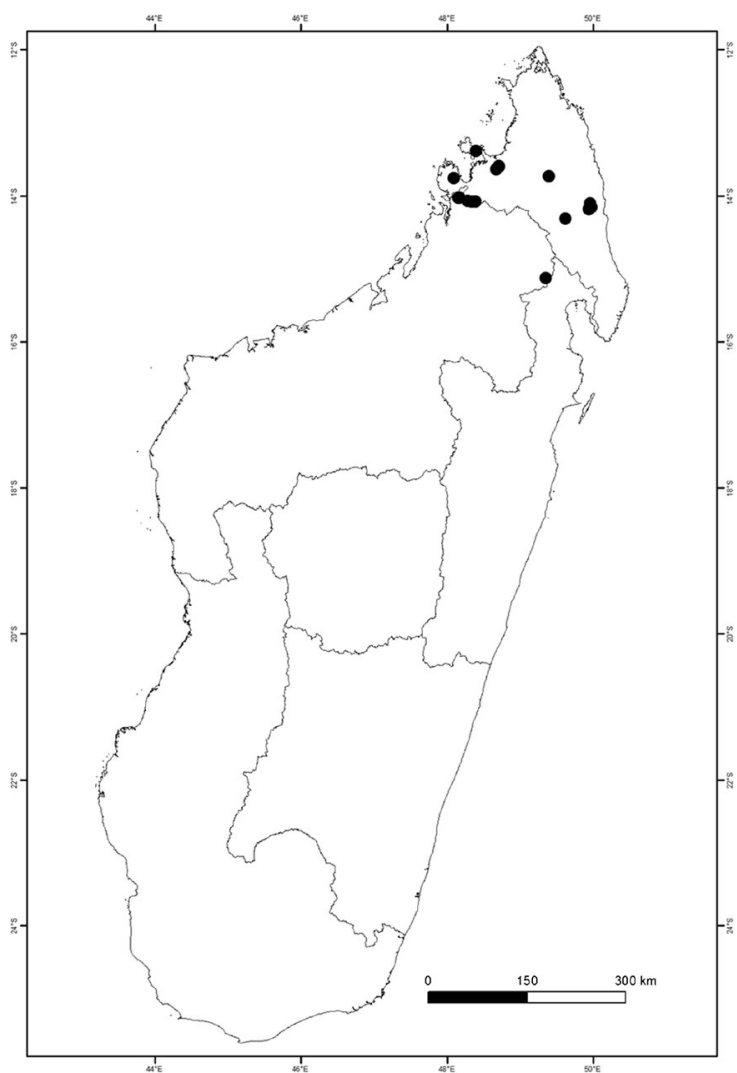

Map 6. Distribution of Phyllanthus sambiranensis in Madagascar.

coriaceous, glabrous on both sides, margin entire, hyaline for $1 / 5$ of tepal width on each side, veins unbranched. Disc glands 5, lenticular, $0.2-0.3 \mathrm{~mm}$ in diam. Stamens 5, entirely fused into a column; column terete, $0.6-1.2 \times 0.8-1 \mathrm{~mm}$; anthers fused, elongate, apiculate, c. $1.2 \times 0.2-3 \mathrm{~mm}$. Female flowers $2.5-2.9$ $\times 2.8$ - $3 \mathrm{~mm}$. Pedicels $0.5-0.8 \times 0.4-0.5 \mathrm{~mm}$, glabrous. Tepals 5, subequal, elliptic or obovate, $1.8-$ $2.2 \times 1-1.2 \mathrm{~mm}$, round, subcoriaceous, glabrous on both sides, margin entire, hyaline for $1 / 5$ of tepal width on each side, veins unbranched. Disc alternitepalous lobed. Ovary 3-locular, globose, $1.5-2 \times 1.5-2 \mathrm{~mm}$, smooth, glabrous. Styles entirely free, deltoid, entire, $0.4-0.5 \mathrm{~mm}$ long; stigmas obtuse. Fruits (only young fruits seen) depressed globose, c. $2.8 \times 3.2 \mathrm{~mm}$; fruiting pedicels c. $2 \times 0.5 \mathrm{~mm}$; tepals $2-2.2 \times 1-$ $1.2 \mathrm{~mm}$. Fig. 5 .

DISTRIBUTION. Endemic to Madagascar. Eastern floristic domain. Map 7.

SPECIMENS EXAMINED. MADAGASCAR. Antsiranana: Marojejy, track between campsite 2 and campsite 3, $14^{\circ} 25^{\prime} 41^{\prime \prime S}, 49^{\circ} 45^{\prime} 36^{\prime \prime S}$, alt. 983 m, 9 Nov. 2010, Andrinaivoravelona et al. 378 (K, TAN); Loky Manambato, $13^{\circ} 12^{\prime} 18^{\prime \prime S}, 49^{\circ} 32^{\prime} 36^{\prime \prime S}$, alt. 1099 m, 28 Nov. 2004, Gautier et al. 4776 (G, TEF); Sommet Est du massif de Marojejy, Ouest de Manantenina, affluent de Lokoho, alt. 1850 - 2137 m, Dec. 1948, Humbert 22761 (P); Valley of Lokoho, Mont Beondroka, $\mathrm{N}$ of Maroambihy, alt. 1400 - 1450 m, Humbert 23608 (P, lectoparatype); valley of Ambatoharanana, bassin inferior of Antsahaberoka, alt. 1600 - 1800 m, 15 - 25 Nov. 1959, Humbert Eं Saboureau 31819 (P); Marojejy, $\mathrm{N}$ of Mandena, $14^{\circ} 26^{\prime} \mathrm{S}, 49^{\circ} 46^{\prime} \mathrm{E}$, alt. $800-1300 \mathrm{~m}, 3$ Dec. 1989, Miller $\mathcal{E}$ Randrianasolo 4656 (P); Marojejy, track to the summit, $14^{\circ} 2632^{\prime \prime S}, 49^{\circ} 44^{\prime} 24^{\prime \prime E}$, alt. 1585 m, 16 Oct. 2011, Rakotoarinivo et al. 588 (K, TAN); Marojejy Andapa, campement III, 11 Oct. 1988, Rakotozafy E Raharilala 2266 (TAN); Andapa, Marovato, $14^{\circ} 35^{\prime} 29^{\prime \prime S}, 49^{\circ} 39^{\prime} 15^{\prime \prime E}$, alt. $710 \mathrm{~m}, 4$ Feb. 2014, Onjalalaina et al. 254 (K, TAN); Marojejy summit, 14²6'57"S, 4943'56"E, alt. $2145 \mathrm{~m}, 11$ May 2015, Rabarijaona et al. 1021 (K, TAN); Marojejy, 14²5'33"S, 4942'46"E, alt. 1510 m, 20 Nov. 2003, Ranaivojaona 565 (MO, TAN); Marojejy, 14²6'20"S, 4942'15"E, 20 Nov. 2003, Ranaivojaona 568 (MO, TAN).

HABITAT. Margin of humid forests. Altitude $800-1510 \mathrm{~m}$. CONSERVATION STATUS. Phyllanthus marojejiensis has an AOO of $24 \mathrm{~km}^{2}$, and an EOO of $838.21 \mathrm{~km}^{2}$, two locations are known for this species and one of them is inside the Marojejy protected area. This species is threatened by habitat loss due to forest logging, illegal mining and annual fire. It is therefore rated as Endangered (EN B2ab(iii,v)).

8. Phyllanthus lichenisilvae (Leandri) Petra Hoffm. E McPherson (2003: 308). Glochidion lichenisilvae Leandri (1937 : 29). Type: Madagascar. Mont Tsaratanana, 2000 m, silve à lichens, April 1921, Perrier de la Bâthie 16414 (holotype P!).

Monoecious shrubs, 3 - $4 \mathrm{~m}$ high. Branching pinnatiform; orthotropic branches terete, striate, glabrous. Cataphylls triangular, $0.8-1 \times 0.3-0.5 \mathrm{~mm}$, coriaceous, entire, glabrous. Cataphyllary stipules triangular, $0.5-0.8 \times 0.3-0.5 \mathrm{~mm}$, coriaceous, entire, glabrous. Plagiotropic branches solitary, terete or compressed, 3 - $8 \mathrm{~cm}$ long, 0.4 - $0.5 \mathrm{~mm}$ diam., striate, glabrous. Stipules persistent, triangular or subulate, $1-1.2 \times 0.2-0.3 \mathrm{~mm}$, coriaceous, entire, glabrous. Leaves 15 - 33 per branch. Internodes 1 $3 \mathrm{~mm}$ long. Petioles absent. Leaf blades elliptic, asymmetrical, $4-7 \times 2.5-3 \mathrm{~mm}, 1.6-2.3 \times$ longer than wide, attenuate at the base, obtuse, acute or mucronulate at the apex, subcoriaceous to coriaceous, glabrous on both sides; midvein indistinct adaxially, prominent abaxially; secondary veins $3-4$ pairs, flattened or invisible adaxially, prominent abaxially. Inflorescences unisexual, solitary, male inflorescences on proximal and female inflorescences on distal part of 


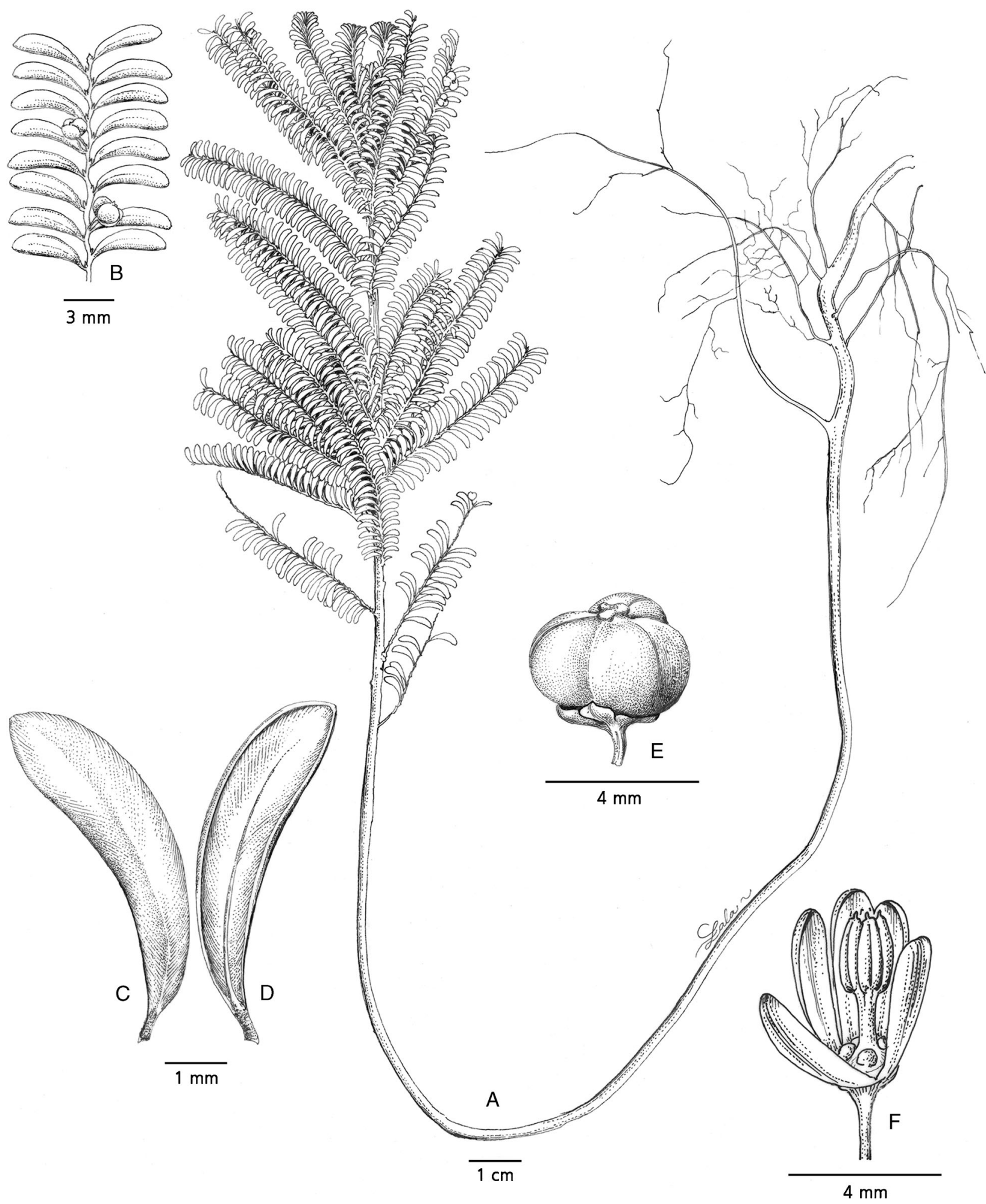

Fig. 5. Phyllanthus marojejiensis (R. Ranaivojaona 565, TAN). A habit; B plagiotropic branch; C leaf (adaxial surface); D leaf (abaxial surface); $E$ fruit; $F$ male flower. DRAWN BY R. L. ANDRIAMIARISOA.

plagiotropic branches. Bracts triangular grouped in a cluster of $5-7$ mainly sterile bracts in male inflorescences, elliptic, $0.5-0.6 \times 0.4 \mathrm{~mm}$, erose, glabrous. Male flowers $1.5-2.3 \times 1-1.5 \mathrm{~mm}$. Pedicels $1.5-2 \times 0.4-0.5 \mathrm{~mm}$, glabrous. Tepals 5 , subequal, oblong or obovate, $1.5-2.3 \times 0.8-1.2 \mathrm{~mm}$, round, 


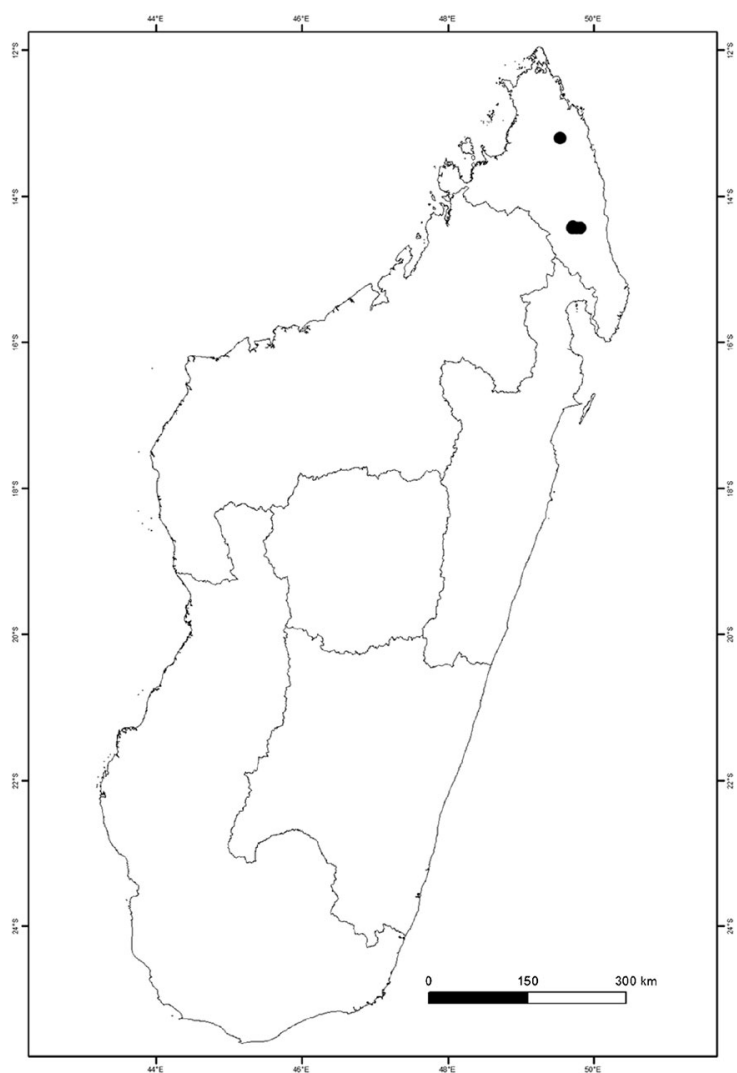

Map 7. Distribution of Phyllanthus marojejiensis in Madagascar.

coriaceous, glabrous on both sides, margins entire, hyaline for $1 / 5$ of tepal width on each side, veins branched. Disc glands 5, lenticular c. $0.2 \mathrm{~mm}$ in diam. Stamens $4-5$ with a central apiculum $($ c. $1 \times 0.3 \mathrm{~mm})$, entirely fused into a column; column terete, c. $0.6 \times$ $0.5 \mathrm{~mm}$; anthers fused, elongate. Female flowers 2 $3.5 \times 2.5-4 \mathrm{~mm}$. Pedicels c. $1.2 \times 0.4 \mathrm{~mm}$, glabrous Tepals 5, unequal, obovate, $2.8-3.5 \times 1.4-1.6 \mathrm{~mm}$, obtuse, coriaceous, glabrous on both sides, margins entire, hyaline for $1 /{ }_{6}$ of tepal width on each side, veins branched. Disc annular pentagonal. Ovary 3-locular, globose, $1.8-2 \times$ c. $2 \mathrm{~mm}$, smooth or slightly rugose, glabrous. Styles entirely free, deltoid, slightly bifid, 0.3 - 0.4 mm long; stigmas obtuse. Fruits (only young fruits seen), globose.; tepals $2.8-3.5 \times 1.4-1.6 \mathrm{~mm}$.

DISTRIBUTION. Endemic to Madagascar. Central floristic domain. Map 8.

SPECIMENS EXAMINED. MADAGASCAR. Antsiranana: Manongarivo, Bekolosy, $14^{\circ} 02^{\prime} \mathrm{S}, 48^{\circ} 18^{\prime} \mathrm{E}$, alt. $1490 \mathrm{~m}$, 16 Nov. 1994, Gautier Eं Dereleth 2568 (G, P); Marojejy massif, alt. 2000 - 2137 m, Nov. 1972, Morat 4074 (P); Mont Tsaratanana, 2000 m, silve à lichens, April 1921, Perrier de la Bâthie 16414 (P).

HABITAT. Margins of humid forests, open humid forests. Altitude $1490-2137 \mathrm{~m}$.

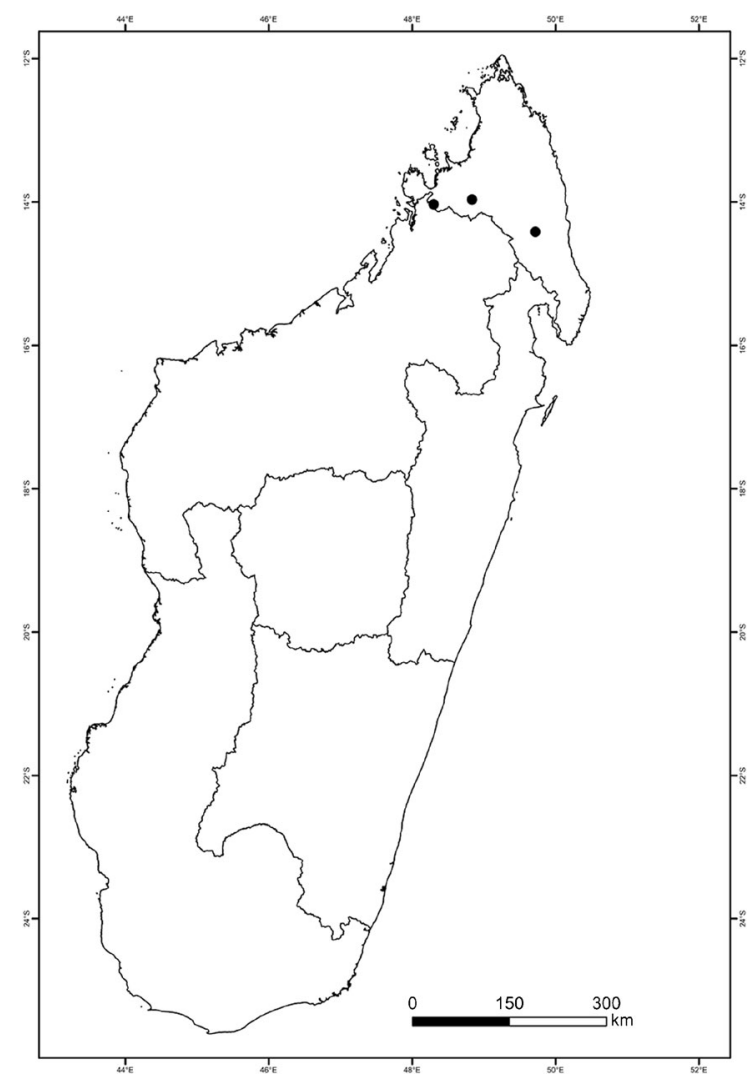

Map 8. Distribution of Phyllanthus lichenisilvae in Madagascar.

CONSERVATION STATUS. Phyllanthus lichenisilvae has an EOO of $1804 \mathrm{~km}^{2}$, AOO of $12 \mathrm{~km}^{2}$ and two locations in three protected areas (Manongarivo, Marojejy and Tsaratanana). It is rated as Endangered (EN Blab(i,ii,iii,iv) + 2ab(i,ii,iii,iv)).

9. Phyllanthus ankaratrae (Leandri) Petra Hoffm. Eं McPherson (2003: 308). Glochidion ankaratrae Leandri (1957: 214). Type: Madagascar. Environs Ambositra, forêt de Ranomena, July 1929, Humbert E S Swingle 4845 (holotype P!).

Monoecious shrubs, c. $1 \mathrm{~m}$ high. Branching pinnatiform; orthotropic branches terete, rugose or striate, glabrous. Cataphylls triangular, $1-1.2 \times 0.4-$ $0.5 \mathrm{~mm}$, coriaceous, entire, glabrous. Cataphyllary stipules triangular, $0.7-1 \times 0.3-0.5 \mathrm{~mm}$, coriaceous, entire, glabrous. Plagiotropic branches solitary or paired, terete or compressed, $3-9 \mathrm{~cm}$ long, $0.4-$ $0.6 \mathrm{~mm}$ in diam., striate, glabrous. Stipules persistent, triangular, $0.5-1 \times 0.2-0.3 \mathrm{~mm}$, slightly coriaceous, entire, glabrous. Leaves 12 - 33 per branch. Internodes $1-2.5 \mathrm{~mm}$ long. Petioles absent. Leaf blades elliptic to lanceolate, asymmetrical, $5-11 \times 2.5-4.5 \mathrm{~mm}, 2-2.4$ $\times$ longer than wide, attenuate at the base, acute at the 
apex, membranous to chartaceous, glabrous on both sides; midvein prominent or flattened adaxially, prominent abaxially; secondary veins $3-5$ pairs, indistinct adaxially, flattened or slightly prominent abaxially. Inflorescences unisexual, solitary, male inflorescences on proximal and female inflorescences on distal part of plagiotropic branches. Bracts triangular, in a cluster of 4 - 7 mainly sterile bracts in male inflorescences, obovate, $0.4-0.5 \times 0.2-0.4 \mathrm{~mm}$, slightly erose, glabrous. Male flowers $2-3 \times 2-2.5 \mathrm{~mm}$. Pedicels $1-$ $1.3 \times 0.3-0.4 \mathrm{~mm}$, glabrous. Tepals 5, subequal, oblong or obovate, $2-3 \times 1-1.2 \mathrm{~mm}$, rounded, membranous, glabrous on both sides, margins entire, hyaline for $1 / 6$ of tepal width on each side, veins branched. Disc glands 5, lenticular, $0.1-0.2 \times 0.1-0.2$ $\mathrm{mm}$. Stamens $3-5$, entirely fused into a column; column terete, $0.6-1.3 \times 0.5-0.8 \mathrm{~mm}$; anthers fused, elongate, not apiculate, and with different lengths, 1 $1.3 \times 0.2 \mathrm{~mm}$. Female flowers c. $1.5 \times 1.5 \mathrm{~mm}$. Pedicels $1-1.3 \times 0.4-0.5 \mathrm{~mm}$, glabrous. Tepals 6 , subequal, elliptic or obovate, $1.3-1.5 \times 1-1.2 \mathrm{~mm}$, rounded, membranous, glabrous on both sides, margins entire, hyaline for $1 / 6$ of tepal width on each side, veins branched. Disc extremely reduced or absent. Ovary 3locular, sub-globose, $1-1.3 \times 1-1.4 \mathrm{~mm}$, smooth, glabrous. Styles entirely free, deltoid, slightly bifid, 0.2 - $0.3 \mathrm{~mm}$ high; stigmas obtuse. Fruits (only young fruits seen) sub-globose; tepals $1.4-1.5 \times 1-1.6 \mathrm{~mm}$. Fig. 6.

DISTRIBUTION. Endemic to Madagascar. Central floristic domain. Map 9.

SPECIMENS EXAMINED. MADAGASCAR. Antananarivo: track to Tsiazompaniry to Andapa, Ambohimiadana Andramasina, June 1961, Capuron 20293SF (TEF); Fianarantsoa: Mont Tsaratanana, $2000 \mathrm{~m}$, silve à lichens, April 1921, Perrier de la Bathie 16414 (P); Ambalavao, Ambalamanasy, Ambondrombe, 21 $51^{\circ} \mathrm{S}$ 047¹6'E, 26 May 2004, Rakotonasolo 802 (MO, TAN).

HABITAT. Open humid forest, forest boundaries. Altitude 1300 - 1700 m.

CONSERVATION STATUS. Phyllanthus ankaratrae has an AOO of $12 \mathrm{~km}^{2}$, and an EOO of $4161.15 \mathrm{~km}^{2}$. Three locations and three subpopulations are known for this species. All of the subpopulations occur outside of protected areas. The species is threatened by habitat loss due to annual fire and forest logging and illegal mining. It is therefore assessed as Endangered (EN Blab(iii, v) + 2ab(iii, v)).

\section{Incertae sedis}

1. Phyllanthus analamerae Leandri (1957: 225). Type: Madagascar. Antsiranana, plateau calcaire Analamera, Jan. 1938, Humbert 19151 (holotype P!).
Dioecious shrubs or a small tree, 1 - $2 \mathrm{~m}$ high. Branching pinnatiform; orthotropic branches compressed, rugose or flaky, glabrous. Cataphylls triangular, $1.8-2.5 \times 0.8-1 \mathrm{~mm}$, coriaceous, entire, glabrous. Cataphyllary stipules triangular, $1.5-2.5 \times$ 0.6 - $1 \mathrm{~mm}$, subcoriaceaous, entire, glabrous. Brachyblasts terete ending by a cluster of $5-8$ bracts, 5-10 mm long; bracts of brachyblast triangular, $1-2$ $\times 0.3-0.5 \mathrm{~mm}$, coriaceous, entire. Plagiotropic branches solitary or fascicled by two, terete, 1.5 $8 \mathrm{~cm}$ long, $0.5-0.8 \mathrm{~mm}$ in diam., pubescent. Stipules persistent, linear, $1-3 \times 0.2-0.4 \mathrm{~mm}$, membranous, entire, with hyaline margin, glabrous. Leaves $3-7$ per branch. Internodes $3-10 \mathrm{~mm}$ long. Petioles terete, 1 $-2 \times 0.4-0.6 \mathrm{~mm}$, glabrous or pubescent. Leaf blades ovate, rarely elliptic, $10-32 \times 7-22 \mathrm{~mm}, 1.4-1.5 \times$ longer than wide, obtuse to truncate at the base, obtuse to acute at the apex, chartaceous or membranous, glabrous on both sides or with thin hairs adaxially; midvein prominent on both sides, pubescent on upper side; secondary veins $2-3$ pairs, flattened adaxially, slightly prominent abaxially. Male and female flower not seen. Information about female flowers deduced from fruit characters. Pedicels glabrous. Tepals 5, equal, obovate or oblong, subcoriaceous, glabrous, margins entire, hyaline for $1 / 2$ of tepal width on each side, veins unbranched. Disc lobed, 0.4 - $0.6 \mathrm{~mm}$ thick, verrucose. Ovary 3-locular, globose or depressed globose, smooth, glabrous. Styles entirely free or fused at the base, bifid for $2 / 3$ their length, terete, $1-1.5 \mathrm{~mm}$ long; stigmas obtuse or acute. Fruits globose or depressed globose, $3-6 \times 4-8 \mathrm{~mm}$; fruiting pedicels $20-30 \times 0.3-0.4 \mathrm{~mm}$; tepals $1.6-$ $2.5 \times 0.8-1.2 \mathrm{~mm}$; columella $2.5-4 \times 0.8-1 \mathrm{~mm}$. Seeds $1.7-1.9 \times 1.2-1.5 \mathrm{~mm}$, with about $10-20$ thin undulating and longitudinal striae.

RECOGNITION. This species is similar to members of subgenus Anisonemoides by the capsular fruits but differs from them by the long brachyblasts (vs brachyblasts absent), the seed coat with undulating and longitudinal striae (vs thinly striated or smooth). DISTRIBUTION. Endemic to Madagascar. Western floristic domain. Map 10.

SPECIMENS EXAMINED. MADAGASCAR. Antsiranana: Ankarana, Tsingy, NE Mahamasina, 12 $50^{\prime} 47^{\prime \prime S}$, $49^{\circ} 6^{\prime} 18^{\prime \prime E}$, alt. $82 \mathrm{~m}, 16$ Jan. 2002, De Block et al. 1255 (BR, K, MO, TAN); plateau calcaire Analamera, Jan. 1938, Humbert 19151 (P). Mahajanga: Tsingy of Namoroka, $16^{\circ} 27^{\prime} S, 45^{\circ} 19^{\prime} \mathrm{E}$, alt. 140 m, 29 Sept. 1983, Du Puy et al. 383 (K, TAN); Ambilobe, Antsahatompy, Antasahatompy forest, $13^{\circ} 37^{\prime} 05.4^{\prime \prime} \mathrm{S}, 50^{\circ} 00^{\prime} 20^{\prime \prime} \mathrm{E}$, alt. 96 m, 3 April 2013, Ralimanana et al. 1767 (K, MO, P, TAN).

HABITAT. Dry forests. Altitude $140 \mathrm{~m}$. 


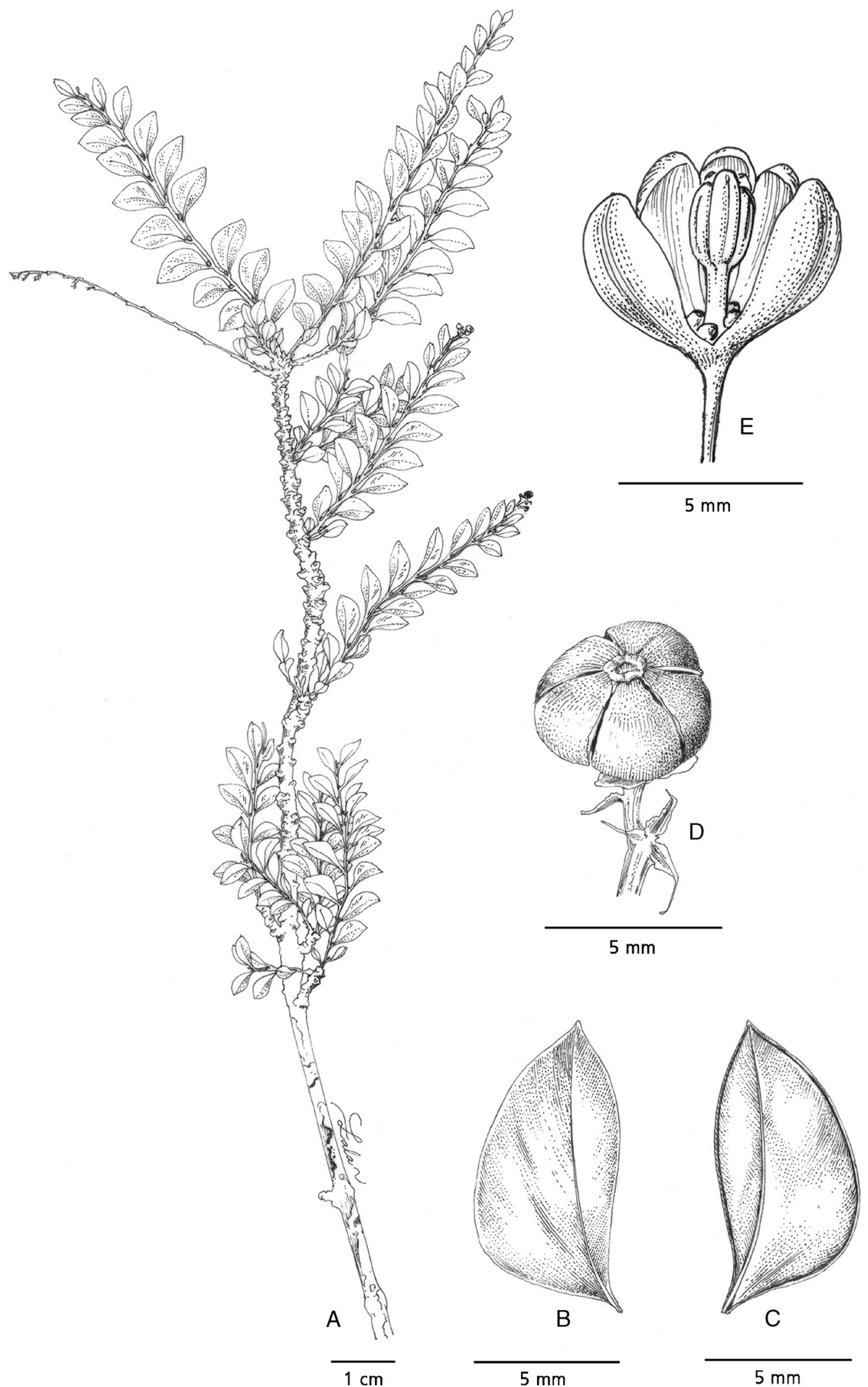

Fig. 6. Phyllanthus ankaratrae (F. Rakotonasolo 802, TAN; SF 20293, TEF). A habit; B leaf (adaxial surface); C leaf (abaxial surface); D fruit; E male flower. DRAWN BY R. L. ANDRIAMIARISOA. 


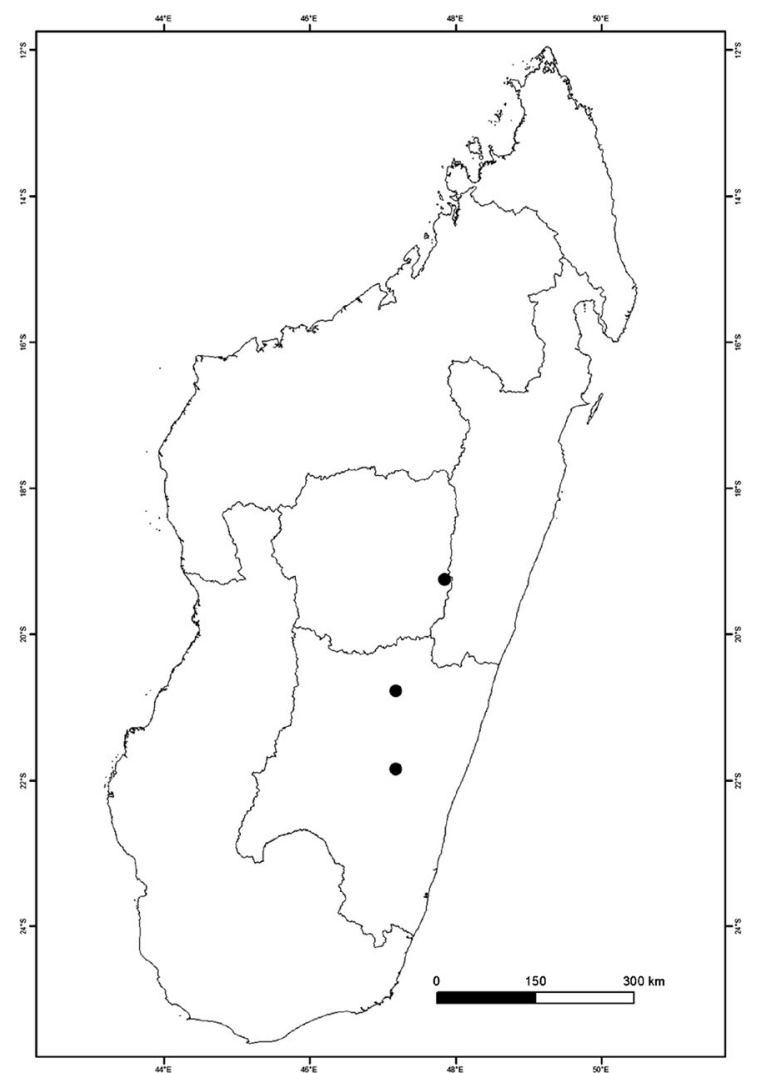

Map 9. Distribution of Phyllanthus ankaratrae in Madagascar.

CONSERVATION STATUS. Phyllanthus analamerae has an AOO of $16 \mathrm{~km}^{2}$, four subpopulations are known for this species and two of them occur in Ankarana and Namoroka protected areas. The subpopulations outside of the protected areas are threatened by habitat loss due to mining exploitation and forest logging. It is therefore rated as Endangered (EN B2ab(ii,iii,iv)).

2. Phyllanthus coluteoides Müll. Arg. (Müller 1866: 335). Type: Madagascar. Antsiranana, Baie de Diego, Dec. 1848, Boivin 2664 (holotype P!).

Dioecious shrubs, 1 - 2 m high. Branching pinnatiform; orthotropic branches terete, rugose, glabrous. Cataphylls triangular, $1-1.2 \times 0.5 \mathrm{~mm}$, erose, glabrous. Cataphyllary stipules triangular, $0.7-1 \times 0.5 \mathrm{~mm}$, coriaceous, entire, glabrous. Brachyblasts terete, 6 $9 \mathrm{~mm}$ long. Plagiotropic branches solitary or fascicled by $2,4.5-9 \mathrm{~cm}$ long, $0.3 \mathrm{~mm}$ in diam., striate, glabrous. Stipules triangular or linear, $0.4-7 \times 0.2 \mathrm{~mm}$, coriaceous, erose, glabrous. Leaves $3-7$ per branch. Internodes $2-5 \mathrm{~mm}$ long. Petioles terete, c. $1 \times 0.3$ $\mathrm{mm}$, glabrous. Leaf blades suborbicular or ovate, $6-10$ $\times 5-10.3 \mathrm{~mm}, 0.9-1.2 \times$ longer than wide, rounded or obtuse at the base, rounded or obtuse at the apex,

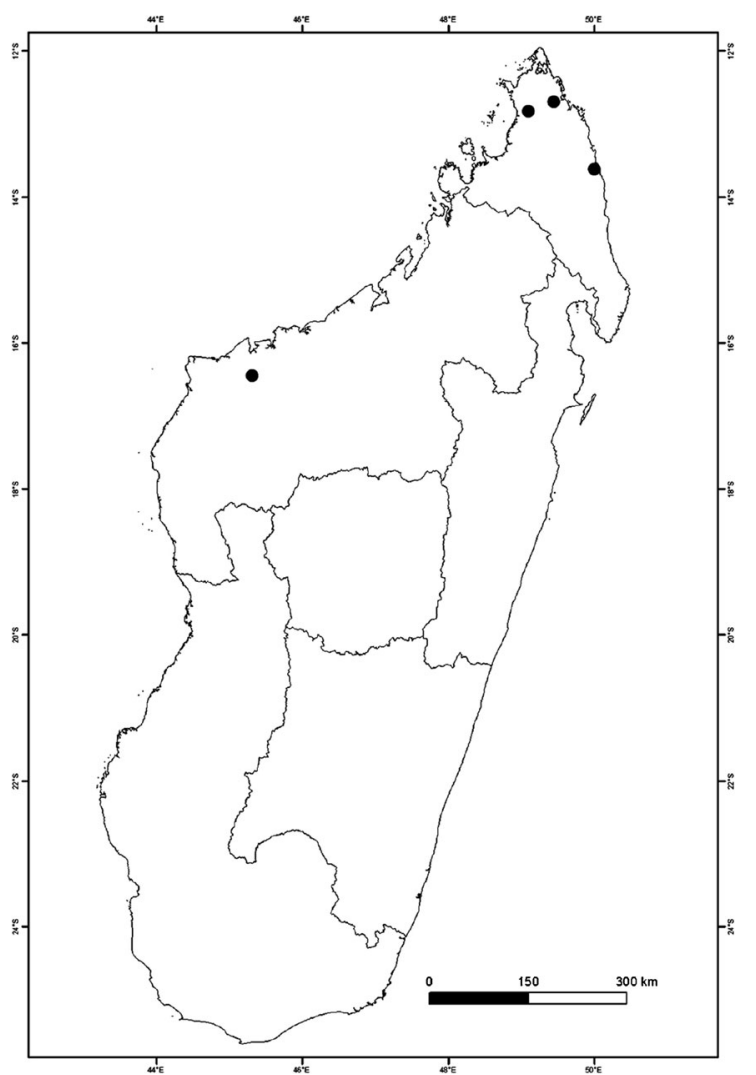

Map 10. Distribution of Phyllanthus analamerae in Madagascar.

membranous, glabrous; midvein prominent on both sides, secondary veins $4-5$ pairs, prominent on both sides. Male and female flowers not seen. Female flowers information derived from fruits characters. Bracts triangular, $0.7 \times 0.4 \mathrm{~mm}$, glabrous. Pedicels glabrous. Tepals 6, inequal, obovate, membranous, glabrous on both sides, veins branched. Disc lobed. Ovary 3-locular, globose, smooth, glabrous; styles, bifid for $1 / 3$ their length, fused at the base; stigmas obtuse or acute. Fruits depressed globose, $4-5 \times 5-6 \mathrm{~mm}$; fruiting pedicels $20-30 \mathrm{~mm}$ long; tepals c. $1.6 \times 0.6$ $\mathrm{mm}$; columella $2-3 \times 0.4-0.6 \mathrm{~mm}$. Seeds $1.8-2.5 \times$ $1.5-2 \mathrm{~mm}$, smooth.

RECOGNITION. Phyllanthus coluteoides is similar to the members of subgenus Anisonemoides by the style bifid and fused at the base and the capsular fruit, the lack of male flower information makes the placement doubtful.

DISTRIBUTION. Endemic to Madagascar. Western floristic domain.

SPECIMENS EXAMINED. MADAGASCAR. Antsiranana: Baie de Diego, Dec. 1848, Boivin 2664 (P). Toliara: 26 Oct. 1940, Decary 16139 (P). Juan de Nova Island: Tsingy, $17^{\circ} 3^{\prime} 40^{\prime \prime} \mathrm{S}, 42^{\circ} 43^{\prime} 29^{\prime \prime} \mathrm{E}$, alt. $5 \mathrm{~m}, 16$ March 2013, Hivert et al. 546 (CBNM); Perrier de la Bathie 13788 (P). HABITAT. Dry forest, thickets. 
CONSERVATION STATUS. Phyllanthus coluteoides has a very restricted distribution and is known from three locations in the northern and south-western domains of Madagascar. The AOO is $12 \mathrm{~km}^{2}$. Its habitat is declining due to fire, wood harvesting for charcoal production, conversion to pasture and agricultural activities. The three threatened locations are outside protected areas and are thought to be small and declining. This species is rated as Endangered (EN B2ab(ii, iii, iv)).

3. Phyllanthus vergens Baill. (Baillon 1892: 225). Type: Madagascar. Sambirano, station de Vavatobe, Feb. 1880, Hildebrandt 3328 (holotype P!; isotype, K!).

Apparently dioecious shrubs or subshrubs. Branching pinnatiform; orthotropic branches terete, rugose or striate, glabrous. Cataphylls triangular, $0.5-1 \times 0.4-0.8 \mathrm{~mm}$, coriaceous entire or erose, glabrous. Cataphyllary stipules triangular, $0.3-1 \times 0.4-0.8 \mathrm{~mm}$, coriaceous, entire, glabrous. Plagiotropic branches fasciculate by $2-4$, terete, $4-10 \mathrm{~cm}$ long, $0.5-1 \mathrm{~mm}$ in diam., striate, glabrous. Stipules persistent or caducous, triangular, $0.3-0.4 \times 0.2-$ $0.3 \mathrm{~mm}$, coriaceous, entire, glabrous. Leaves 5 - 12 per branch. Internodes $4-13 \mathrm{~mm}$ long. Petioles semiterete or flattened, $2-5 \times 0.7-1 \mathrm{~mm}$, glabrous. Leaf blades elliptic, $13-43 \times 6-19 \mathrm{~mm}, 2.1-2.3 \times$ longer than wide, attenuate at the base, acute at the apex, coriaceous, revolute, glabrous on both sides; midvein impressed adaxially, prominent abaxially; secondary veins $3-6$ pairs, impressed adaxially, prominent abaxially. Inflorescences unisexual, consisting of 2 -5 male flowers, on leafless branches borne at the base of plagiotropic branches. Bracts triangular, $0.2-0.4 \times 0.2-0.3$ $\mathrm{mm}$, entire or erose, glabrous. Male flowers $0.8-1 \mathrm{~mm}$ high, $0.6-1 \mathrm{~mm}$ in diam. Pedicels $0.7-1 \times 0.2-0.3 \mathrm{~mm}$, glabrous. Tepals 5, unequal, elliptic, $0.7-1 \times 0.4-0.6 \mathrm{~mm}$, obtuse, coriaceous, margins entire, hyaline for $1 / 3-1 / 2$ of tepal width on each side, glabrous on both sides, veins branched. Disc glands 5, globose, $0.1-0.2 \times 0.1-0.2 \mathrm{~mm}$. Stamens 3 ( -5 ), free; filaments terete, $0.4-0.6 \times 0.1-0.2$ $\mathrm{mm}$; anthers free, ovoid, $0.1-0.2 \mathrm{~mm}$ long. Female flowers and fruit not seen.

DISTRIBUTION. Endemic to Madagascar. Sambirano floristic domain.

SPECIMENS EXAMINED. Species only known from the type.

HABITAT. Humid forest.

CONSERVATION STATUS. This species is only known from the 1892 type collection despite several expeditions to the area where it was collected. In addition, this area is seriously threatened by human activities such as habitat destruction by shifting agriculture. We believe that this species is very rare, and therefore it is rated as Critically endangered (CR B2ab(i, ii, iii, iv, iv)).

\section{Acknowledgements}

We wish to thank the staff of the following herbaria for allowing us to consult specimens and for their assistance: The Royal Botanic Gardens, Kew (K), Muséum d'Histoire Naturelle, Paris (P), Parc Botanique et Zoologique de Tsimbazaza (TAN) and the Direction de la Recherche Forestière et Piscicole, Antananarivo (TEF). We gratefully acknowledge the Direction Générale des Forêts (DGF) and the Madagascar National Parks (MNP) for providing us authorisation and permits to undertake research within protected and unprotected areas in Madagascar. We also thank Gill Challen for assistance at Kew, L. Rabarivola and H. Razanajatovo (KMCC) for producing the maps, R. L. Andriamiarisoa for the drawings. Special thanks go to the Friends of Kew, the BenthamMoxon Trust and the Royal Botanic Gardens, Kew, who generously supported this study.

Open Access This article is licensed under a Creative Commons Attribution 4.0 International License, which permits use, sharing, adaptation, distribution and reproduction in any medium or format, as long as you give appropriate credit to the original author(s) and the source, provide a link to the Creative Commons licence, and indicate if changes were made. The images or other third party material in this article are included in the article's Creative Commons licence, unless indicated otherwise in a credit line to the material. If material is not included in the article's Creative Commons licence and your intended use is not permitted by statutory regulation or exceeds the permitted use, you will need to obtain permission directly from the copyright holder. To view a copy of this licence, visit http://creativecommons.org/ licenses/by/4.0/.

\section{References}

Brunel, J. F. (1987). Sur le genre Phyllanthus L. et quelques genres voisins de la Tribu des Phyllantheae Dumort. (Euphorbiaceae, Phyllantheae) en Afrique intertropicale et à Madagascar. Thèse doctorale, Université Louis Pasteur, Strasbourg.

Baillon, H. (1892). In: A. Grandidier, Histoire Physique, Naturelle et politique de. Madagascar 2: 225. Paris.

Hoffmann, P. \& McPherson, G. (2003). Transfer of Madagascan Glochidion to Phyllanthus. Novon 13: 307-310.

, Kathriarachchi, H. \& Wurdack, K. J. (2006). A phylogenetic classification of Phyllanthaceae (Malpighiales; Euphorbiaceae sensu lato). Kew Bull. 61: $37-53$.

Humbert, H. (ed.) (1965). Flore de Madagascar et des Comores, 111ème famille. Firmin-Didot, Paris. 
IUCN (2012). IUCN Red list Categories and Criteria : Version 3.1, second edition. IUCN Species Survival Commission, Gland.

Kathriarachchi, H., Samuel, R., Hoffmann P., Mlinarec, J., Wurdack, K. J., Ralimanana, H., Stuessy, T. F. \& Chase, M. W. (2006). Phylogenetics of tribe Phyllantheae (Phyllanthaceae; Euphorbiaceae sensu lato) based on nrITS and plastid matK DNA sequence data. Amer. J. Bot. 93 (4): 637 - 655.

Kato, M., Takimura, A. \& Kawakita, A. (2003). An obligate pollination mutualism and reciprocal diversification in the tree genus Glochidion (Euphorbiaceae). Proc. Nat. Acad. Sci. USA 100: 5264 5267.

\& Kawakita, M. (2017a). Ecological reserach monograph. Obligate pollination mutualism. Springer, Japan.

\& _ (2017b). Biology of the obligate pollination Mutualism. In M. Kato \& A. Kawakita (eds), Obligate Pollination Mutualism: p. 63 - 80. Springer, Japan.

Kawakita, A. \& Kato, M. (2004a). Evolution of obligate pollination mutualism in New Caledonian Phyllanthus (Euphorbiaceae). Amer. J. Bot. 91: 410 - 415. \& (2004b). Obligate pollination mutualism in Breynia (Phyllanthaceae): further documentation of pollination mutualism involving Epicephala moths (Gracillariidae). Amer. J. Bot. 91: 1319 - 1325.

, \& _ (2006). Assessment of the diversity and species specificity of the mutualistic association between Epicephala moths and Glochidion trees. Molec. Ecol. 15: 3567 - 3581.

, \& _ (2009). Repeated independent evolution of obligate pollination mutualism in the Phyllantheae-Epicephala association. Proc. Roy. Soc. London, Ser. B, Biol. Sci. 276: 417 - 426.

Leandri, J. (1934). Espèces et localités nouvelles d'Euphorbiacées d'Afrique et Madagascar. Bull. Soc. Bot. France 81: 449 - 454.
(1935). Catalogue des plantes de Madagascar, Euphorbiaceae. G. Pitot \& Cie, Académie Malgache, Tananarive.

(1937). Contribution à l'étude des Euphorbiacées de Madagascar. Notul. Syst. Paris 6: 11 - 35.

(1957). Notes systématiques sur les Euphorbiacées, Phyllanthus de Madagascar. Mém. Inst. Sci. Madagascar 8: 205 - 234.

(1958). Euphorbiaceae I (Phyllanthoideae). In. H. Humbert (ed.), Flore de Madagascar et des Comores, 111ème famille. Firmin-Didot, Paris.

Müller, J. (1866). Euphorbiaceae. In: A. De Candolle (ed.), Prodromus Systematis Naturalis Regni Vegetabilis 15 (2): 189 - 1286. Victor Masson, Paris.

Ralimanana, H. \& Hoffmann, P. (2011). Taxonomic revision of Phyllanthus (Phyllanthaceae) in Madagascar and the Comoro Islands I: synopsis and subgenera Isocladus, Betsileani, Kirganelia and Tenellanthus. Kew Bull. 66: 331 - 365.

\& Rajeriarison, C. (2013). Taxonomic revision of Phyllanthus (Phyllanthaceae) in Madagascar and the Comoro Islands III: subgenera Swartziani, Afroswartziani and Emblica. Kew Bull. 68: 535 - 558.

\& _ (2014). Taxonomic revision of Phyllanthus (Phyllanthaceae) in Madagascar and the Comoro Islands II: subgenera Anisonemoides (Jean F. Brunel) Ralim. \& Petra Hoffm., stat. nov and Menarda Comm. ex A. Juss.) Ralim. \& Petra Hoffm., stat. nov. Adansonia sér. 3, 36 (2): 265 - 301.

Webster, G. L. (1994). Synopsis of the genera and suprageneric taxa of Euphorbiaceae. Ann. Missouri Bot. Gard. 81 (1): 33 - 144.

\section{Publisher's Note}

Springer Nature remains neutral with regard to jurisdictional claims in published maps and institutional affiliations. 